\title{
Autonomous transport and splitting of a droplet on an open surface
}

\author{
Imdad Uddin Chowdhury $\odot,{ }^{1}$ Pallab Sinha Mahapatra $\odot,{ }^{1, *}$ Ashis Kumar Sen $\odot,{ }^{1}$ \\ Arvind Pattamatta, ${ }^{2}$ and Manish K. Tiwari ${ }^{3,4, \dagger}$ \\ ${ }^{1}$ Micro Nano Bio Fluidics Group, Department of Mechanical Engineering, \\ Indian Institute of Technology Madras, 600 036, India \\ ${ }^{2}$ Department of Mechanical Engineering, Indian Institute of Technology Madras, 600 036, India \\ ${ }^{3}$ Nanoengineered Systems Laboratory, UCL, London, WC1E 7JE, United Kingdom \\ ${ }^{4}$ Wellcome/EPSRC Centre for Interventional and Surgical Sciences, UCL, London, \\ W1W 7TS, United Kingdom
}

(Received 22 February 2021; accepted 8 September 2021; published 27 September 2021)

\begin{abstract}
Pumpless transport of droplets on open surfaces has gained significant attention because of its applications starting from vapor condensation to Lab-on-a-Chip systems. Mixing two droplets on open surfaces can be carried out quickly by using wettability patterning. However, it is quite challenging to split a droplet in the absence of external stimuli because of the interfacial energy of the droplet. Here, we demonstrate a standalone power-free technique for transport and splitting of droplets on open surfaces using continuous wettability gradients. A droplet moves continuously from a low to a high wettability region on the wettability-gradient surface. A Y-shaped wettability-gradient track - laid on a superhydrophobic background - is used to investigate the dynamics of the splitting process. A three-dimensional phase-field Cahn-Hilliard model for interfaces and the Navier-Stokes equations for transport are employed and solved numerically using the finite element method. Numerical results are used to decipher the motion and splitting of droplet at the $\mathrm{Y}$ junction using the principle of energy conservation. It is observed that droplet splitting depends on the configuration of the $\mathrm{Y}$ junction; droplets split faster for the superhydrophobic wedge angle of $90^{\circ}$ and the splitting ratio (ratio of the sizes of daughter droplets) depends on the widths of the $\mathrm{Y}$ branches. A critical branch-width ratio $\left(\frac{w_{2}}{w_{1}}=0.79\right)$ is identified below which the droplet does not split and moves towards the branch of higher width and settles there. The present study provides the required theoretical underpinnings to achieve autonomous transport and splitting of droplets on open surfaces, which has clear potential for applications in Lab-on-a-Chip devices.
\end{abstract}

DOI: 10.1103/PhysRevFluids.6.094003

\section{INTRODUCTION}

Open surface droplet manipulation on engineered surfaces has gained a lot of attention recently and has the potential to spawn a number of surface fluidics devices that may have a transformative impact on micro and nanofluidics. This is exemplified by open surface liquid transport, mixing, metering, splitting, etc. and has applications in Lab-on-a-Chip (LOC) systems [1,2], point-of-care (POC) diagnostics [3], microfluidic devices [4-9], spray cooling [10], etc. Passive droplet migration on a surface using wettability difference is demonstrated by Yuan et al. [11]. Open surface passive liquid mixing was demonstrated by Morrissette et al. [2]. They showed liquid transport and mixing

\footnotetext{
*pallab@iitm.ac.in

†m.tiwari@ucl.ac.uk
} 
by using wettability patterning. In the absence of external force, droplet splitting is difficult to achieve as compared with mixing because of the interfacial energy of the droplet.

Passive droplet manipulation on an open surface using a wettability gradient has gained a lot of interest because of its nonintrusive nature. Various research groups have performed experiments and numerical analyses of droplet manipulation on wettability-gradient surfaces [12-14]. Droplets move on a surface with wettability gradients because of the curvature difference between the front and back of the droplet. This curvature difference creates a net driving force along the direction of droplet transport, so the droplet moves. In our previous work, we performed a numerical analysis of droplet transport on different wettability-gradient surfaces [15], and we identified that the net driving force on a droplet is a maximum for a surface having a gradient of wettability from superhydrophobic to superhydrophilic. Experimentally, it is possible to create such wettability-gradient surfaces [16]. Liu et al. [16] demonstrated a technique of preparing a wettability-gradient surface on a silicon wafer from superhydrophobic (contact angle $\approx 166^{\circ}$ ) to superhydrophilic (contact angle $\approx 15.5^{\circ}$ ). In the present work we used a surface with an almost similar wettability gradient.

Droplet splitting on an open surface resembles the droplet generation technique on a microchannel: in both cases, the fluid interface has to be ruptured. Droplet generation in a microchannel of $\mathrm{Y}$ and T-junction geometries are studied extensively by Ushikubo et al. [17] and Carlson et al. [18]. They observed that the fluids and the interfacial properties play a major role in breaking the interface. There are various active droplet-splitting techniques, such as electric-field-mediated droplet control [19], electrowetting [20,21], using acoustic waves [22,23], using thermal actuation [24], and using magnetic fields [25,26]. Samiei et al. [27] performed a systematic analysis of a geometry-based unequal-droplet splitting in digital microfluidics. They developed an operator for unequal-droplet splitting by geometrical modification of electrodes in digital microfluidics (DMF) platforms. Using this operator, a droplet dispensed from a reservoir can be divided unequally. Although there are a number of active droplet-splitting techniques available in the literature, to the best of our knowledge, there is a paucity of studies on passive open surface droplet-splitting techniques. The open surface platform is advantageous for the splitting of droplets because of its accessibility, ease of fabrication, and adaptability. Song et al. [28] created a superhydrophobic strip on a hydrophilic surface and demonstrated splitting. They used Y-shaped patterns and placed the droplet at the junction of the superhydrophobic strips, which split the droplet into three parts. The volume ratios of the split droplet were controlled by manipulating the position of the droplet placement. The droplet could be split into a greater number of mini droplets by increasing the number of superhydrophobic strips. Berry et al. [29] showed a passive open surface droplet-splitting technique driven by capillary flow. They created a T-shaped bypass open channel and added a carrier fluid for easy transport of the droplet. Although they showed a controlled droplet-splitting technique by using an open channel on the surface, their approach required a carrier fluid to carry the droplet and fabrication of open channels with bypass junctions, which is challenging.

There are some relevant works in the field. For example, the study on droplet running uphill from Whitesides et al. [30], the recent work on jumping or trampolining drops on surface nanostructures [31,32] and on bouncing drops driven by Marangoni stress [33]. Our previous work [15] focused on transporting a droplet on a wettability-confined gradient surface with the primary objective of finding the optimum wettability confinement. However in this work, we mainly focus on the splitting dynamics of a droplet. In microfluidic devices, LOC systems, and the point-of-care (POC) diagnostic systems, often it is required to split droplets a certain distance from the position where the droplets are dispensed. In this work, we proposed an optimum wettability pattern that serves this purpose in a passive manner. Although passive droplet transport on an open surface has been widely studied in the past, the combination of the passive transport and splitting of a droplet together on an open surface platform is not explored yet. To achieve passive droplet displacement, we created a gradient of wettability on a surface, and, to split the moving droplet, we used wettability patterning by creating a Y-shaped wettability-gradient track laid on a superhydrophobic background. With this design, the droplet could be divided into two equal or unequal parts by manipulating the branch widths of the Y track. The approach extends the applications to open surface droplet metering. The 


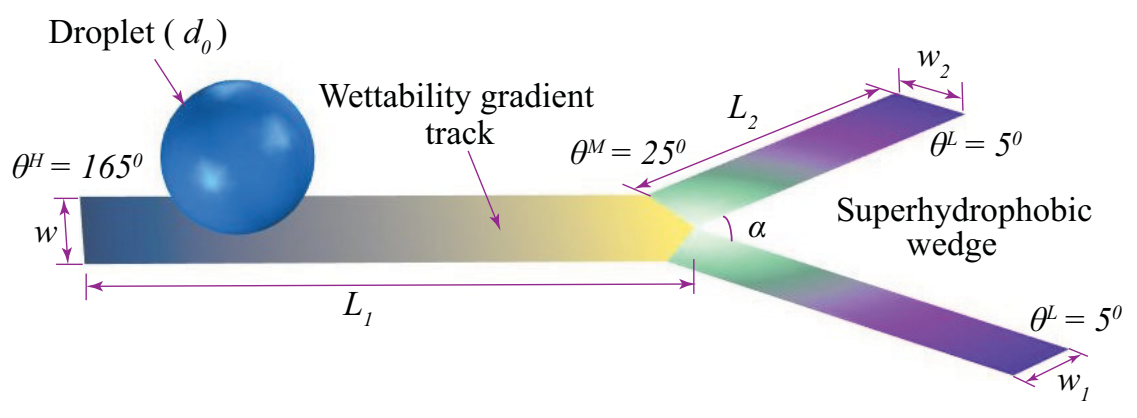

FIG. 1. A droplet of diameter $d_{0}=0.5 \mathrm{~mm}$ is placed on a Y-shaped wettability-gradient track laid on a superhydrophobic background. The superhydrophobic background of the $Y$ track has a contact angle of $170^{\circ}$. The initial position of the droplet is at a distance $d_{0}$ from the left. The base track of the Y-shaped wettability-gradient track has width $w$ and is divided into two branches by a superhydrophobic wedge of wedge angle $\alpha$. The branches of the Y track have widths $w_{1}$ and $w_{2}$. The length of the branches are $L_{2}=1.5 \mathrm{~mm}$ and the length of the base track of $\mathrm{Y}$ is $L_{1}=2 \mathrm{~mm}$. The wettability gradient is linear and the variation of contact angle is from $\theta^{H}=165^{\circ}$ to $\theta^{M}=25^{\circ}$ for length $L_{1}$ and from $\theta^{M}=25^{\circ}$ to $\theta^{L}=5^{\circ}$ for length $L_{2}$. At the bifurcation region, the contact angle is close to $25^{\circ}$, which is represented by yellow color at the base track and the light green at the branches. The transition from yellow to light green is just to distinguish the base track and the branches.

physics of the droplet transport, flow pattern inside the droplet, and the splitting mechanisms have been explained through detailed numerical studies. The physics is explained through scale analysis and with the help of an energy conservation approach. This concept is used here to combine droplet transport and splitting on an open surface platform without creating any channels, which widens the application potential of surface fluidics devices.

\section{MATHEMATICAL FORMULATION}

The problem geometry is shown in Fig. 1. The droplet is placed on a Y-shaped bifurcated wettability-gradient track laid on a superhydrophobic background. The base track (length $L_{1}$ ) and the branches of the Y track (length $L_{2}$ ) have different wettability gradients. For the base track (length $\left.L_{1}\right)$, the variation of the contact angle along the direction of droplet transport is

$$
\theta_{1}(s)=\theta^{H}-\frac{\left(\theta^{H}-\theta^{M}\right)}{L_{1}} s .
$$

The symbol " $s$ " denotes the coordinate along the surface fluidic channel with a wettability gradient. For the branches of the Y track (length $L_{2}$ ), the variation of contact angle along the direction of droplet transport is

$$
\theta_{2}(s)=\theta^{M}-\frac{\left(\theta^{M}-\theta^{L}\right)}{L_{2}} s,
$$

where $\theta^{H}=165^{\circ}, \theta^{M}=25^{\circ}$ and $\theta^{L}=5^{\circ}$ represents the contact angle at the upstream, at the bifurcation point of the $\mathrm{Y}$ track, and at the downstream end of the wettability-gradient track.

The numerical model is implemented in COMSOL Multiphysics [34] using a laminar twophase flow model with the phase-field method $[35,36]$ for tracking the interface. It is assumed that the flow is laminar and incompressible. The system is considered isothermal and at room temperature. The density and viscosity of the droplet and the air are taken as constant. For conservation of mass and momentum, the incompressible continuity and Navier-Stokes equations are 
used [37]:

$$
\begin{gathered}
\nabla \cdot \mathbf{u}=0 \\
\rho \frac{\partial \mathbf{u}}{\partial t}+\rho(\mathbf{u} \cdot \nabla) \mathbf{u}=\nabla \cdot\left[p \mathbf{I}+\mu\left(\nabla \mathbf{u}+\nabla \mathbf{u}^{\mathbf{T}}\right)\right]+\rho \mathbf{g}+\mathbf{F}_{\mathbf{s}}
\end{gathered}
$$

where all the bold quantities are vectors. The average density of the fluid is represented by $\rho$ $\left(\mathrm{kg} / \mathrm{m}^{3}\right)$, the average dynamic viscosity of the fluid is $\mu(\mathrm{Pa} \mathrm{s})$, fluid pressure is $p(\mathrm{~Pa})$, and the velocity vector is denoted by $\mathbf{u}(\mathrm{m} / \mathrm{s})$. The surface tension force is represented by $\mathbf{F}_{\mathrm{s}}\left(\mathrm{N} / \mathrm{m}^{3}\right), I$ represents the identity matrix, and the gravitational constant is denoted by $\mathbf{g}\left(\mathrm{m} / \mathrm{s}^{2}\right)$. The average value of fluid density $\rho$ and the dynamic viscosity $\mu$ is calculated as follows:

$$
\begin{aligned}
& \rho=\rho_{a} V_{f, 1}+\rho_{l} V_{f, 2}, \\
& \mu=\mu_{a} V_{f, 1}+\mu_{l} V_{f, 2},
\end{aligned}
$$

where $\rho_{l}$ and $\mu_{l}$ represent the density and dynamics viscosity of the droplet, respectively, whereas $\rho_{a}$ and $\mu_{a}$ are the density and dynamics viscosity of air, respectively. $V_{f, 1}$ and $V_{f, 2}$ are the fractions of the phase-field function $\phi$, which can be expressed as

$$
\begin{gathered}
V_{f, 1}=\frac{1-\phi}{2}, \\
V_{f, 2}=\frac{1+\phi}{2}, \\
V_{f, 1}+V_{f, 2}=1,
\end{gathered}
$$

where $\phi$ is the phase-field function whose value varies from -1 to 1 across the interface. For air, the value of $\phi$ is -1 , whereas it is 1 for the droplet. From Eqs. (5) and (6), in the droplet domain $\rho=\rho_{l}(\phi=1)$, and $\rho=\rho_{a}(\phi=-1)$ in the air domain.

The phase-field method gives a better alternative for solving multiphase flow situations. The Cahn-Hilliard equation is used to capture the evolution of the interface which is a fourth-order partial differential equation (PDE). In this method, the diffused interface is tracked using a phase-field function $\phi$. The Cahn-Hilliard equation not only convects the interface but also ensures energy conservation of the system. The phase-field function $\phi$ is governed by the following equation [36]:

$$
\frac{\partial(\phi)}{\partial t}+\mathbf{u} \cdot \nabla \phi=\nabla \cdot \chi \nabla G
$$

where the mobility $\chi\left(\mathrm{m}^{3} \mathrm{~s} / \mathrm{kg}\right)$ represents the timescale of diffusion for the Cahn-Hilliard equation. $G$ is the chemical potential, which is calculated as the rate of change of free energy $F$ at the interface $\left(G=\frac{\partial F}{\partial \phi}\right)$. The free energy $F$ is expressed as [37]

$$
F(\phi, \nabla \phi)=\int\left(\frac{1}{2} \lambda^{2}|\nabla \phi|^{2}+K_{0}(\phi)\right) d V,
$$

wherein using $\epsilon_{p f}(m)$ as the liquid-gas interface thickness, we can identify $\frac{1}{2} \epsilon_{p f}{ }^{2}|\nabla \phi|^{2}$ as the interfacial free-energy density and $K_{0}=\frac{\lambda}{4 \epsilon_{p f}{ }^{2}}\left(\phi^{2}-1\right)^{2}$ [37] is the bulk-energy density. To choose the interface thickness $\epsilon_{p f}$ we referred to Wu et al. [38]. They observed that the numerical results vary drastically for Cahn number $\mathrm{Cn}=\frac{\epsilon_{p f}}{d_{0}}>0.15$. Since the variation in the result is very small for $\mathrm{Cn}<0.15$ and we also observed it in our numerical simulation, and thus we have chosen $\mathrm{Cn}=0.15$. The reason behind choosing the highest possible value of $\mathrm{Cn}$ is to reduce the computational expense. For the highest value of $\mathrm{Cn}$, we can get the maximum possible interface thickness $\epsilon_{p f}$ for which the solver works properly without affecting the results. In the present work, we consider the diameter of the droplet $d_{0}$ as $0.5 \mathrm{~mm}$ and thus we have $\epsilon_{p f}=75 \mu \mathrm{m}$. We understand that the chosen interface 
thickness is not realistic, but this is the limitation of the numerical modeling of such sharp interfaces. If we consider a realistic value (thickness on the nm scale) of the interface then the number of grid cells becomes so large that it requires a supercomputer to simulate. Thus, for numerical modeling of such sharp interfaces, researchers use the maximum feasible value of the interface thickness, which generally ranges from 10 to $100 \mu \mathrm{m}$ [38]. The mixing energy density $\lambda(N)$ is related to the interfacial energy of the droplet $\sigma$ as $\sigma=\frac{2 \sqrt{2}}{3} \frac{\lambda}{\epsilon_{p f}}$ [36]. The chemical potential $G$ can be expressed as [37]

$$
G=\frac{\partial F}{\partial \phi}=\lambda\left(-\nabla^{2} \phi+\frac{\phi\left(\phi^{2}-1\right)}{\epsilon_{p f^{2}}}\right) .
$$

In the present case, we decompose the Cahn-Hilliard equation into two second-order PDEs as follows [34,36]:

$$
\begin{gathered}
\frac{\partial(\phi)}{\partial t}+\mathbf{u} \cdot \nabla \phi=\nabla \cdot \frac{\lambda \chi}{\epsilon_{p f^{2}}} \nabla \psi \\
\psi=-\nabla \cdot \epsilon_{p f}^{2} \nabla \phi+\left(\phi^{2}-1\right) \phi+\left(\frac{\epsilon_{p f}^{2}}{\chi}\right) \frac{\partial f}{\partial \phi} .
\end{gathered}
$$

The value of the mobility $\chi$ should be high enough to maintain a constant interface thickness, whereas it should be low enough to damp the convective motion. The mobility $\chi$ is controlled by a mobility-tuning parameter $\beta$. The mobility is calculated as $\chi=\beta \epsilon_{p f}^{2}$ [36]. The parameter $\beta$ should not be too high to avoid excessive diffusion and it should be sufficiently small to maintain a constant interface thickness [34]. A value of $1 \mathrm{~m} \mathrm{~s} / \mathrm{kg}$ is a good starting point for most of the models [34]. We started with $\beta=1 \mathrm{~m} \mathrm{~s} / \mathrm{kg}$ and we found that the solution is insensitive for $\beta \leqslant 0.01 \mathrm{~m} \mathrm{~s} / \mathrm{kg}$. Thus we have considered $\beta=0.01 \mathrm{~m} \mathrm{~s} / \mathrm{kg}$. The external free energy is represented by $f\left(\mathrm{~J} / \mathrm{m}^{3}\right)$. In the present case, the phi derivative of the external free energy, $\frac{\partial f}{\partial \phi}$, is taken as zero. The surface tension force $F_{s}$ is calculated as [34]

$$
\mathbf{F}_{\mathbf{s}}=\left(\frac{\chi}{\epsilon_{p f^{2}}} \psi-\frac{\partial f}{\partial \phi}\right) \nabla \phi .
$$

We considered all the boundaries of the three-dimensional (3D) domain (see Fig. 1) as zero pressure "outlet" boundary conditions except the bottom boundary where wettability patterning is performed. The bottom boundary is called the "wetted wall" where the Navier slip boundary condition is adopted. A negligible hysteresis is considered at the wetted wall. The Navier slip boundary condition enforces a finite tangential velocity at the wetted wall. The tangential velocity component is zero at an extrapolated distance of $\beta$ from the wall. It also enforces the no-penetration boundary condition at the wetted wall as

$$
\mathbf{u} \cdot \hat{\mathbf{n}}_{\text {wall }}=0
$$

where the normal unit vector at the wetted wall is represented by $\hat{\mathbf{n}}_{\text {wall }}$.

\section{NONDIMENSIONAL PARAMETERS, NUMERICAL METHODOLOGY, AND ENERGY BALANCE}

\section{A. Characteristic quantities and nondimensional parameters}

Considering the characteristic length as the diameter of the droplet $d_{0}$, for a droplet in steady-state motion on a wettability-gradient surface, the droplet velocity can be expressed as $\frac{K_{1} \sigma d_{0}}{\mu_{l}} \frac{d \cos \theta}{d x}[39]$. The symbol $K_{1}$ is a constant. We have chosen this steady-state droplet velocity as the characteristic velocity $U_{c}$. Thus, for the linear wettability gradient considered,

$$
U_{c}=\frac{\sigma d_{0}}{L \mu_{l}}\left(\left|\cos \theta^{H}-\cos \theta^{L}\right|\right)
$$


where $L=\left(L_{1}+L_{2}\right)$ is the total physical length of the wettability-gradient track. The characteristic time $T_{c}$ is

$$
T_{c}=\frac{d_{0}}{U_{c}}=\frac{L \mu_{l}}{\sigma\left(\left|\cos \theta^{H}-\cos \theta^{L}\right|\right)} .
$$

The change in droplet surface energy is the driving potential for droplet motion. The characteristic rate of change of surface energy can be expressed as $\dot{E}_{c}=\frac{E_{s o}}{T_{c}}$, with $E_{s o}=\sigma A_{l v}$ being the initial available surface energy of the droplet and $A_{l v}=\pi d_{0}{ }^{2}$.

We solved the Navier-stokes equation by considering both fluids as a single fluid by taking the average of fluid properties across the interface, and $U$ represents the resultant velocity of the fluid. Thus, the nondimensional fluid velocity $U^{*}=\frac{U}{U_{c}}$. However, the average velocity of the droplet $U_{d}$ is calculated as [13]

$$
U_{d}=\frac{\int_{v} \eta(\phi) U d V}{\int_{v} \eta(\phi) d V},
$$

where $\eta(\phi)=1$ for $\phi \geqslant 0$ represents the droplet phase and $\eta(\phi)=0$ for $\phi<0$ represents the air phase. The nondimensional rate of change of energy $\dot{E}^{*}=\frac{\dot{E}}{\dot{E}_{c}}$, where $\dot{E}$ is the rate of energy. The nondimensional time $t^{*}=\frac{t}{T_{c}}$, where $t$ represents the physical time. A nondimensional parameter

$$
\lambda=\left|\frac{\left(\cos \theta^{\text {up }}-\cos \theta^{\text {down }}\right) d_{0}}{L_{i}}\right|
$$

is introduced to characterize the wettability gradient of a surface. The symbol $L_{i}$ denotes the length of the track on which droplet moves. For the base track $L_{i}=L_{1}$ and for the branch track $L_{i}=L_{2}$. The upstream and downstream contact angle of the wettability-gradient surface is $\theta^{\text {up }}$ and $\theta^{\text {down }}$, respectively. For the base track $\theta^{\text {up }}=\theta^{H}$ and $\theta^{\text {down }}=\theta^{M}$, whereas for the branches of the $\mathrm{Y}$ track $\theta^{\text {up }}=\theta^{M}$ and $\theta^{\text {down }}=\theta^{L}$ as shown in Fig. 1.

We defined the nondimensional wetted area of the droplet as $A^{*}=A / A_{0}$, where $A$ is the transient wetted area of the droplet which increases while the droplet moves from the low-wettability region to the high-wettability region, and $A_{0}=\pi d_{0}^{2} / 4$ is the initial projected wetted area of the droplet.

\section{B. Energy conservation}

When a droplet is deposited on a wettability-gradient substrate, the substrate-droplet system tries to attain a minimum-energy state and hence the movement of the droplet. The initially available energy of the droplet includes kinetic energy, gravitational energy, and surface energy. During the movement of the droplet, this available energy decreases continuously and is absorbed by the different forms of dissipations. The droplet-substrate system follows the energy conservation as [40]

$$
\frac{d}{d t}\left(E_{k}+E_{g}+E_{s}\right)+\frac{d}{d t}\left(E_{v}+E_{c l}+E_{\text {film }}\right)=0
$$

where the kinetic energy of the droplet is $E_{k}$, the gravitational energy is $E_{g}$, and the surface energy is $E_{s}$. The dissipation energy has three parts, namely, viscous dissipation $E_{v}$, contact line dissipation $E_{c l}$, and the precursor film dissipation $E_{\text {film }}$ [40]. As reported by Attane et al. [40], $E_{\text {film }}$ is mostly negligible for partial wetting. Since in the present study more than $80 \%$ of the droplet remains in the partial wetting state, we have not considered $E_{\text {film }}$. The Bond number $\mathrm{B}_{0} \ll 1$ so $E_{g}$ is negligible compared with the other available energies $E_{s}$ and $E_{k}$. The rate of change of kinetic energy of the droplet $\dot{E}_{k}$ is expressed as

$$
\dot{E}_{k}=\frac{d}{d t}\left(\sum \frac{1}{2} m U_{d}^{2}\right),
$$


where $m$ represents the mass of the droplet and $U_{d}$ represents the average velocity of the droplet. The symbol $\sum$ denotes the summation of all $U_{d}$ inside the droplet at a particular time instant. To calculate the surface energy of the droplet $E_{s}$, a finite change in surface energy $d E_{s}$ while the droplet transports can be calculated as $d E_{s}=\sigma_{l v} d A_{l v}+\sigma_{s v} d A_{s v}+\sigma_{s l} d A_{s l}$ [41], where the surface tensions of the three phases are represented by $\sigma_{l v}, \sigma_{s v}$, and $\sigma_{s l}$ with $l, v$, and $s$ corresponding to the liquid, vapor, and solid, respectively. In this work we used $\sigma$ as a liquid vapor surface tension of the liquid in place of $\sigma_{l v}$. The area of different phases are represented by $A_{l v}, A_{s v}$, and $A_{s l}$. Because $d A_{s v}=$ $-d A_{s l}$ and with the help of Young's equation, $d E_{s}$ can be written as $d E_{s}=\sigma_{l v}\left(d A_{l v}-\cos \theta_{\mathrm{avg}} d A_{s l}\right)$, where the average contact angle of the droplet is calculated as $\theta_{\text {avg }}=\left(\theta_{f}+\theta_{r}\right) / 2$ with $\theta_{f}$ and $\theta_{r}$ corresponding to the front and back contact angle of the droplet. Hence, the rate of change of surface energy of the droplet, $\dot{E}_{s}$, is [41]

$$
\dot{E}_{s}=\frac{d}{d t}\left[\sigma_{l v}\left(A_{l v}-\cos \theta_{\text {avg }} A_{s l}\right)\right]
$$

The dynamics of the contact line can be better explained by kinetic molecular theory and hydrodynamic theory. Since this is not really in the scope of our work, we simplified the contact line dynamics for the calculation of contact line dissipation. The dissipation at the contact line $E_{c l}$ is due to the motion of the fluid molecule at the three-phase contact region. $E_{c l}$ is present for a smooth solid surface [40]. The magnitude of $E_{c l}$ depends on the surface heterogeneity. $E_{c l}$ is more for a rough surface as compared with the purely smooth solid surface. The rate of change of contact line dissipation, $\dot{E}_{c l}$, is calculated as [40]

$$
\dot{E_{c l}}=\int_{\Gamma} \mu_{f} \epsilon_{p f}\left(\frac{d \phi}{d t}\right)^{2} d \Gamma,
$$

where $\epsilon_{p f}$ is the interface thickness, and $\mu_{f}$ is the friction factor whose value depends on the surface wettability and the viscosity of the droplet [42]. The value of $\mu_{f}$ varies from 0.1 to 1 [42]. As we considered a smooth solid surface, so it is appropriate to have a low value of $\mu_{f}$ so that the friction is minimum. Thus, in the present study we have taken the value of $\mu_{f}$ as 0.1 [42]. The symbol $\Gamma$ represents the perimeter of the droplet footprint. The viscous dissipation $E_{v}$ is in the core of the droplet, which is due to the internal viscous flow. The rate of change of viscous dissipation $\dot{E}_{v}$ is expressed as [40]

$$
\begin{aligned}
\dot{E}_{v}= & \int_{v} 2 \mu_{l}\left[\left(\frac{d u}{d x}\right)^{2}+\left(\frac{d v}{d y}\right)^{2}+\left(\frac{d w}{d z}\right)^{2}\right]+\mu_{l}\left[\left(\frac{d u}{d x}+\frac{d v}{d y}\right)^{2}\right. \\
& \left.+\left(\frac{d v}{d z}+\frac{d w}{d y}\right)^{2}+\left(\frac{d w}{d x}+\frac{d u}{d z}\right)^{2}\right] d V
\end{aligned}
$$

\section{Grid-independent test and time stepping}

A grid-independent test was performed for the droplet transport on a Y-shaped bifurcated wettability-gradient track. The droplet diameter is taken as $0.5 \mathrm{~mm}$ and the length of the base track $L_{1}=2 \mathrm{~mm}$, the length of the branch track $L_{2}=1.5 \mathrm{~mm}$, the width of the branch tracks $w_{1}=w_{2}=0.3 \mathrm{~mm}$, and the width of the base track $w=0.6 \mathrm{~mm}$, as shown in the Fig. $1 . A^{*}$ increases sharply up to $t^{*} \approx 300$, after which the increment in $A^{*}$ slows down. The variation of $A^{*}$ clearly becomes negligible for $h_{1} \leqslant 0.056 d_{0}$, as shown in Fig. 2 . Thus, the independent grid size is considered as $0.056 d_{0}$.

Pseudo-time-stepping is used to solve the governing equations. A fictitious time derivative serves the purpose of pseudo-time-stepping as

$$
\frac{\mathbf{u}-\mathbf{u}^{*}}{\Delta t}+(\mathbf{u} \cdot \nabla) \mathbf{u}=\frac{1}{\rho} \nabla \cdot[-p \mathbf{I}]+\nabla \cdot\left[v\left(\nabla \mathbf{u}+(\nabla \mathbf{u})^{\mathbf{T}}\right)\right]+\mathbf{g}+\frac{\mathbf{F}_{\mathbf{s}}}{\rho},
$$




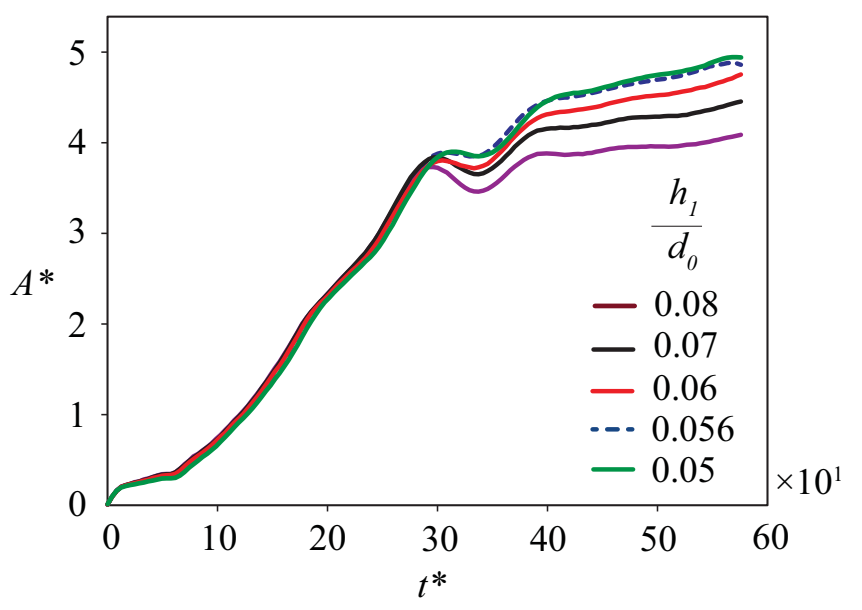

FIG. 2. The variation of the nondimensional wetted area of the droplet $A^{*}$ with nondimensional time $t^{*}$ plotted for different element sizes $h_{1}$ to get the independent grid size. The dotted line represents the independent grid.

where $\mathbf{u}^{*}$ is the guess velocity. The average kinematic viscosity is denoted by $v=\frac{\mu}{\rho}$ and the pseudotime-step by $\Delta t$, which is calculated as

$$
\Delta t=R \frac{h_{1}}{|\mathbf{u}|}
$$

where $h_{1}$ is the grid size and the Courant-Friedrichs-Lewy (CFL) number is represented by $R$. A small $\Delta t$ corresponds to a small CFL number. A proportional integral derivative (PID) regulator controls the CFL number. At each time instant, the solver starts with a small CFL number and sends the feedback to the solver using the PID regulator to check the convergence of the solver. The feedback loop continues until the solution converges.

\section{Validation}

To validate the solver, we have chosen the droplet transport on a linear wettability-gradient surface as reported by Ahmadlouydarab and Feng [43]. A tetraethylene-glycol droplet of $1 \mathrm{~mm}$ diameter having a density of $1130 \mathrm{~kg} / \mathrm{m}^{3}$, a viscosity of $0.05 \mathrm{Pas}$, and a surface tension of $0.046 \mathrm{~N} / \mathrm{m}$ at room temperature [12], is placed on a wettability-gradient surface with an upstream contact angle of $92.3^{\circ}$ and a downstream contact angle of $23^{\circ}$. The variation of the contact angle is linear. The length of the wettability-gradient track is taken as $L=6.67 \mathrm{~mm}$. For simplicity, the density of the surrounding medium is considered to be the same as the droplet density. The viscosity ratio is defined as $N=\frac{\mu_{l}}{\mu_{a}}$. We have performed the validation for two viscosity ratios $N=1$ and $N=50$. The thickness of the interface is taken as $\epsilon_{p f}=20.3 \mu \mathrm{m}$ [43] and the grid size is taken as $h_{1}=5 \mu \mathrm{m}$ such that there are four grid cells across the interface. The results are independent of the chosen grid size. The mobility is taken as $\chi=1.387 \times 10^{-9} \mathrm{~m}^{3} \mathrm{~s} / \mathrm{kg}$ [43]. Thus the mobility-tuning parameter turns out to be $3.467 \mathrm{~m} \mathrm{~s} / \mathrm{kg}$. The slip length is considered as $20 \mu \mathrm{m}$ [43]. Nondimensional center position of the droplet is defined as $x^{*}=\frac{x}{L}$ and, the capillary number of the droplet is $\mathrm{Ca}=\frac{\mu_{l} U_{d}}{\sigma}$. The average velocity of the droplet is represented by $U_{d}$. Figure 3 shows the variation of $\mathrm{Ca}$ while the droplet moves on the wettability-gradient surface. The validation shows a very good match (within 3\%) with the numerical result of Ahmadlouydarab and Feng [43]. 


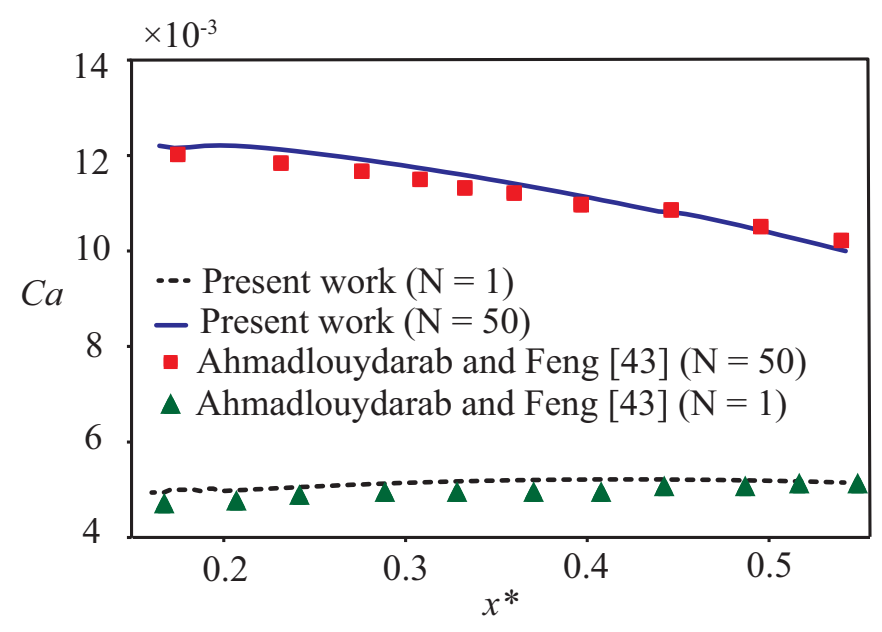

FIG. 3. The variation of the capillary number of the droplet $\mathrm{Ca}$ while it transports on a wettability-gradient surface. The red square and green triangle correspond to the Ca of Ahmadlouydarab and Feng [43]. The blue and black lines represent the present simulated Ca.

\section{RESULTS AND DISCUSSION}

\section{A. Droplet-splitting dynamics}

Under a wettability gradient, the motion of the droplet is due to the curvature difference between the front and back half of the droplet [12]. Here we examine how the droplet splits into two equal or unequal parts during the transport of the droplet depending on the branch width of the $\mathrm{Y}$ track.

Figure 4 shows the splitting dynamics of the droplet for the branch width ratio $w_{2} / w_{1}=1$ and $\alpha=90^{\circ}$. Up to $t^{*}=161$, the droplet moves smoothly on the base track due to the increase in wettability from left to right (length $L_{1}$ ). The wettability gradient of the base track is $\lambda=0.47$. During the transport, the droplet spreads continuously, and the velocity of the droplet increases sharply up to $t^{*}=161$. For $t^{*} \geqslant 161$, the droplet encounters the superhydrophobic wedge. The superhydrophobic wedge has very low wettability and it offers resistance to droplet motion. The
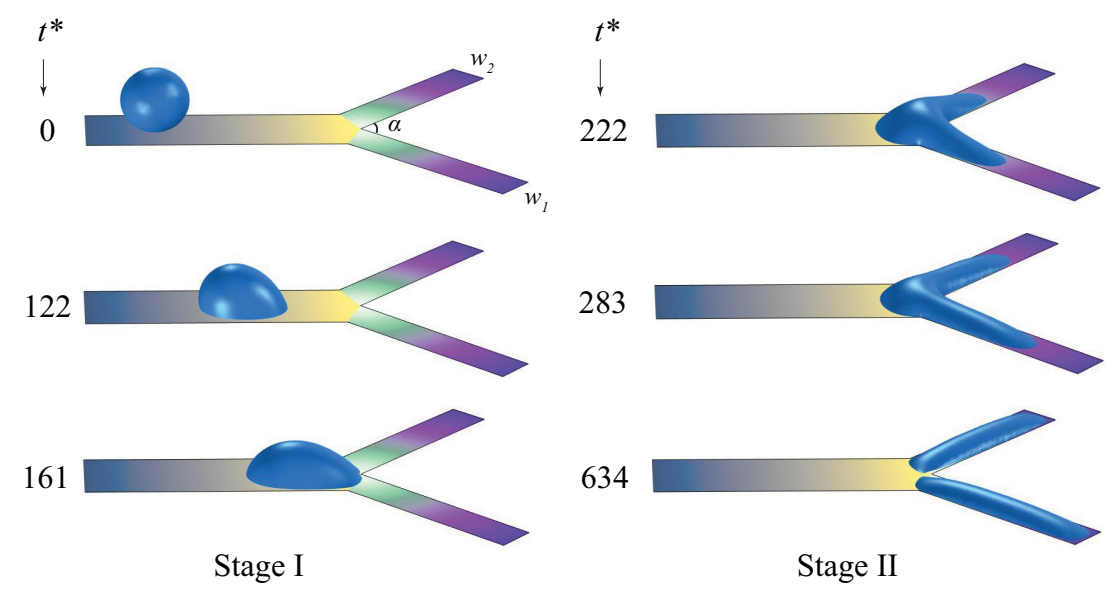

FIG. 4. Droplet splitting on a Y-shaped bifurcated wettability-gradient track with the branch width ratio $w_{2} / w_{1}=1$ and the superhydrophobic wedge angle $\alpha=90^{\circ}$. 
(a)

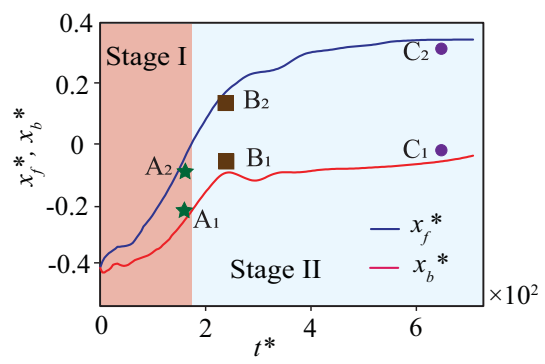

(c)

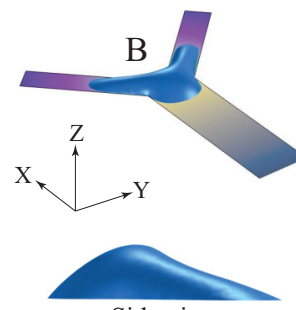

Side view

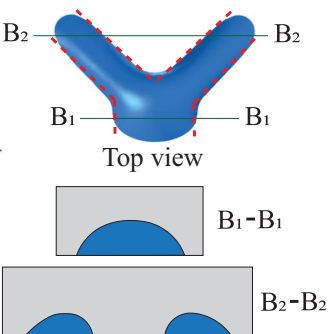

(b)
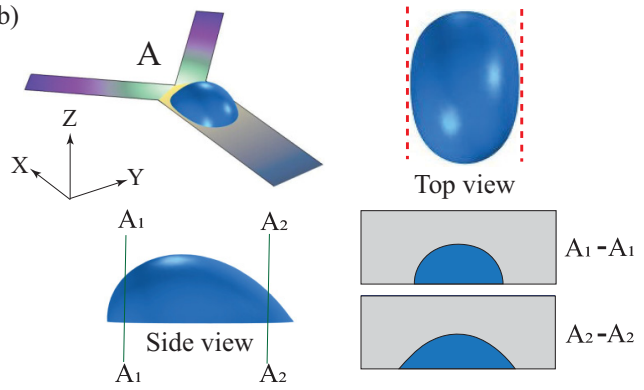

(d)

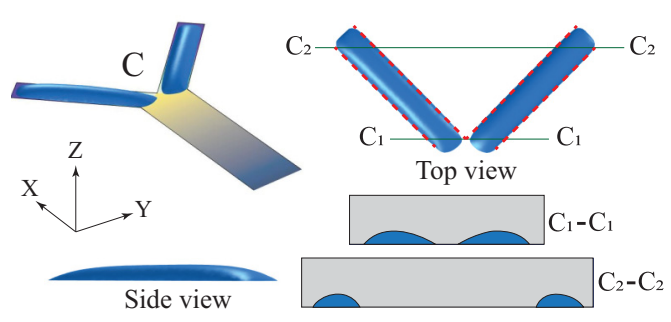

FIG. 5. (a) Variation of the nondimensional front and back position of the droplet $x_{f}{ }^{*}$ and $x_{b}{ }^{*}$, respectively, with $t^{*}$. The droplet shape evolution with closeup looks (side view, top view, and sectional profile) of droplet spreading: (b) position A (end of Stage I), (c) position B (initial phase of Stage II), and (d) position C (end of Stage II) for $w_{2} / w_{1}=1$ and the wedge angle $\alpha=90^{\circ}$.

branches of the Y track have very high wettability (hydrophilic zone) and a wettability gradient, which creates a driving force. The net effect is splitting of the droplet along two branches in the $\mathrm{Y}$ track. The splitting occurs from $t^{*}=161$ to $t^{*}=634$, dividing the droplet into two parts. Since we have considered $w_{2}=w_{1}$, the droplet splits into two equal volumes. We divide the whole transport and splitting process of the droplet into two stages. The smooth droplet transport on the base track of Y is denoted Stage I (up to $t^{*}=161$ ) and the splitting of the droplet at the superhydrophobic wedge is denoted Stage II $\left(t^{*} \geqslant 161\right)$.

Figure 5(a) plots the nondimensional front $\left(x_{f}{ }^{*}\right)$ and back $\left(x_{b}{ }^{*}\right)$ positions of the droplet. Both $x_{f}{ }^{*}$ and $x_{b}{ }^{*}$ increase sharply in Stage I and slow down significantly in Stage II. In Stage I, the droplet is on the base track, which has a higher wettability-gradient $\lambda$ as compared with the branches of the $Y$ track. Points A1, A2 (end of Stage I), B1, B2 (start of Stage II), and C1 and C2 (end of Stage II) are the positions of the droplet at which the zoomed views of the droplet is presented in Figs. 5(b)-5(d). Figure 5(b) shows a zoomed view of the droplet shape at $t^{*}=150$ (position A), where the droplet is in the base track $(\lambda=0.47)$. It is observed that the distribution of droplet volume is lower in the front part of the droplet as compared with the back part (see the sectional profiles A1-A1 and A2-A2, and the side view of the droplet at position A). This is mainly due to the wettability contrast on the surface. The curvature difference between the droplet front and back, which drives the droplet, is also clearly apparent. Figure 5(c) shows the closeup of the droplet shape at $t^{*}=238$, where the droplet is exposed to the superhydrophobic wedge. The cross-sectional profiles (B1-B1 and B2-B2) and the side view of the droplet at position B suggest that a larger fraction of the droplet volume is on the back side as compared with the front. Figure 5(d) shows the droplet shape at the end of the splitting $\left(t^{*}=634\right)$. Because of the equal branch width, the volumes in the branches are identical. As the branches of Y track have a low wettability gradient $(\lambda=0.03)$ as compared with the base track $(\lambda=0.47)$, the curvature difference between the back and front part of the droplet at the branches is minimal (see the sectional profiles $\mathrm{C} 1-\mathrm{C} 1$ and $\mathrm{C} 2-\mathrm{C} 2$ and the side view of the droplet at position C). 
(a)

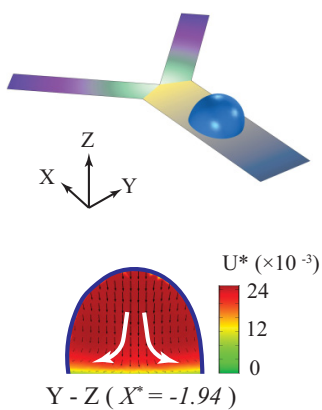

(c)
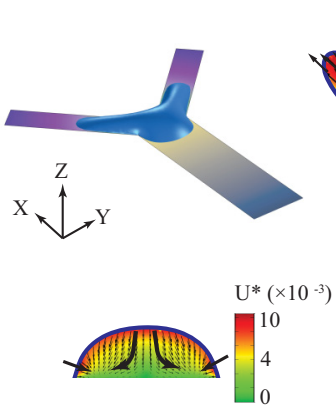

$\mathrm{Y}-\mathrm{Z}\left(X^{*}=-0.2\right)$
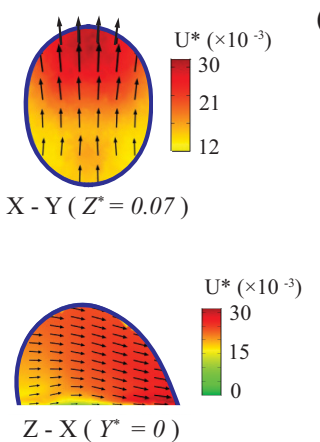

(b)

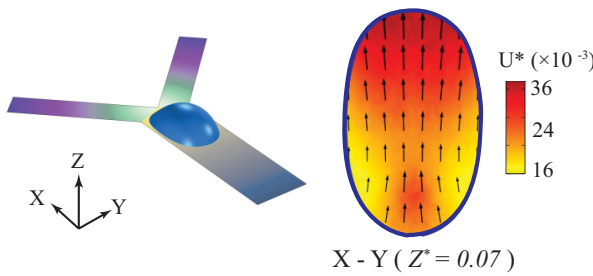

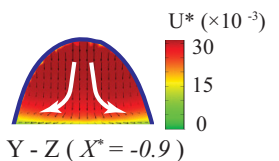

(d)
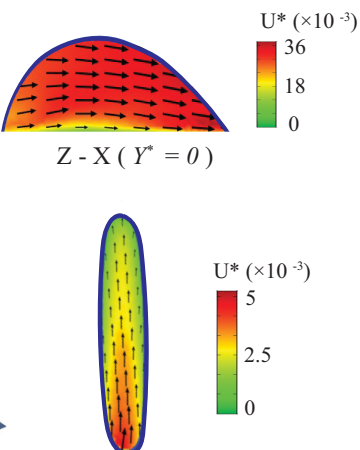

$\mathrm{r}-\mathrm{r}\left(Z^{*}=0.07\right)$
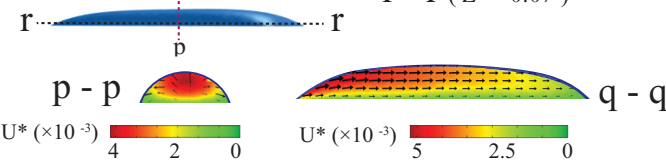

FIG. 6. Velocity contour with overlaying vector plot inside the droplet during transport on a Y-shaped bifurcated wettability-gradient track having a branch-width ratio $w_{2} / w_{1}=1$ and the superhydrophobic wedge angle $\alpha=90^{\circ}$. The reference point $(X, Y, Z)=(0,0,0)$ is the bifurcation point of the Y track. The contours and velocity vectors are plotted on three different cross sections at (a) $t^{*}=112$, (b) $t^{*}=150$, (c) $t^{*}=238$, and (d) $t^{*}=634$. The velocity vectors represent the direction of fluid velocity is shown by black arrows. The droplet interface is shown by the solid blue line.

The splitting dynamics of the droplet during the transport on a Y-shaped bifurcated track can be better understood by the velocity contour with vector plots, as shown in Fig. 6 . The velocity contour and the vectors correspond to the nondimensional fluid velocity $U^{*}$. We consider four different time instants during the droplet transport: $t^{*}=112, t^{*}=150, t^{*}=238$, and $t^{*}=634$, as shown in Figs. 6(a)-6(d), respectively. The velocity data are plotted at three cross sections $X-Y, Y-Z$, and $Z-X$ for $t^{*}=112, t^{*}=150$, and $t^{*}=238$. The cross-section $\mathrm{X}-\mathrm{Y}$ is at a height $Z^{*}=\frac{Z}{d_{0}}=0.07$ away from the substrate while the cross-section $\mathrm{Z}-\mathrm{X}$ is through the reference point $Y^{*}=\frac{Y}{d_{0}}=0$ which bisects the droplet. As the droplet is moving in the $X$ direction, the $Y-Z$ plane which passes through the droplet center varies while the droplet transports. At $t^{*}=112$, the droplet is at the base track and the velocity inside the droplet is nonuniform, as shown in Fig. 6(a). The $X-Y$ and $Z-X$ cross sections suggest that the nondimensional velocity of the fluid $U^{*}$ is more in the front half of the droplet as compared with the back half. This is because of the high wettability of the substrate in the front part of the droplet which leads to higher inertia in the front contact line. The maximum value of $U^{*}$ at $t^{*}=112$ is 0.03 . At $t^{*}=112$, the droplet center is at $X^{*}=\frac{X}{d_{0}}=-1.94$ thus, the $Y-Z$ cross section is taken at $X^{*}=-1.94$ and the vector plot suggests that the fluid moves from droplet top to bottom and finally direct towards the lateral contact line [refer to the $Y-Z$ cross section of Fig. 6(a)]. This movement of the fluid in the vertical direction is due to the continuous height reduction of the droplet top interface while it moves. Figure 6(b) shows the velocity data inside the droplet while the droplet is at $t^{*}=150$. The behavior of the fluid velocity inside the droplet at $t^{*}=150$ is almost 
similar to the fluid velocity at $t^{*}=112$. The maximum value of $U^{*}$ is little bit higher $(=0.036)$ at $t^{*}=150$ as compared with $t^{*}=112$. The droplet at $t^{*}=150$ has higher spreading then at $t^{*}=112$ and thus a higher driving force, which leads to a higher $U^{*}$.

We have taken a time instant of Stage II $\left(t^{*}=238\right)$ to understand the role of fluid velocity during the splitting of the droplet, as shown in Fig. 6(c). Notably, the $X-Y$ cross section suggests four high-velocity patches in the velocity contour $U^{*}$, two are at the back part of the droplet (base track), and the other two patches are at the front part of the droplet (branches). There are four patches in the velocity contour $U^{*}$ [refer to the $X-Y$ cross section of Fig. 6(c)]. The two patches of $U^{*}$ at the branches are due to the tension on the droplet in the direction of droplet transport. In contrast, the other two patches of $U^{*}$ at the base track are due to the obstruction faced by the droplet on a superhydrophobic wedge. The vector plot suggests that the two base track patches of $U^{*}$ are in the opposite direction of droplet transport, and the magnitude is low compared with the front patches [see $X-Y$ cross section of Fig. 6(c)]. The two patches at the base track are due to the combined effect of wettability confinement of the base track end corners at the bifurcation junction and the obstruction of the superhydrophobic wedge. Whereas, the $Z-X$ cross section of Fig. 6(c) shows fluid motion in the opposite direction with a relatively low velocity due to the backward inertia of the droplet after facing the obstruction in the superhydrophobic wedge. Due to the continuous spreading of the droplet, there is a downward movement of the fluid, as shown in cross section $Y-Z$ of Fig. 6(c). The inward movement of fluid at the contact line is because of the wettability confinement at the end corners of the base track in the bifurcation junction.

Finally, we show the velocity plot just after the splitting of the droplet (at $t^{*}=634$ ) in Fig. 6(d). Due to the symmetry in the branches, we concentrate on the velocity data only for one branch. We have taken three cross sections p-p, q-q, and r-r. The cross section r-r is at the height of $Z^{*}=0.07$ away from the substrate. The r-r and q-q cross sections show that the fluid velocity is more at the back end than at the front of the splitting droplet, as the droplet separates at the bifurcation point. The fluid velocity inside the droplet is nonuniform. It is observed that the fluid velocity is maximum at the top of the droplet and decreases to a minimum value near the substrate, as shown in p-p and $\mathrm{q}-\mathrm{q}$ cross sections.

\section{B. Energy balance}

Various forms of energies associated with the droplet motion on a wettability-gradient surface is discussed in Sec. III. The rate of change of contact line dissipation, $\dot{E}_{c l}$, can be simplified as [42]

$$
\dot{E_{c l}}=\int_{\Gamma} \mu_{f} \epsilon_{p f}\left(\frac{d \phi}{d t}\right)^{2} d \Gamma \approx \mu_{f} \epsilon_{p f}\left(\frac{d \phi}{d r}\right)^{2}\left(\frac{d r}{d t}\right)^{2}\left(P_{c} \epsilon_{p f}\right) \approx \mu_{f} P_{c} U_{c l}^{2}
$$

where the perimeter of the contact line is represented by $P_{c}$ which changes during the droplet transport because of the continuous spreading of the droplet and $\Gamma=P_{c} \epsilon_{p f}$. The average contact line velocity is represented by $U_{c l}=\frac{d r}{d t}$. The variation of phase field variable $\phi$ along the radial direction of the droplet is approximated as $\frac{d \phi}{d r} \approx \frac{1}{\epsilon_{p f}}$ [42]. Thus, it is observed from Eq. (27) that contact-line dissipation is not really dependent on the interface thickness $\epsilon_{p f} . \dot{E}_{c l}$ is scaled as $\mu_{f} d_{0} U_{c l}{ }^{2}$ [refer to Eq. (27)], whereas the viscous dissipation $\dot{E}_{v}$ is scaled as $\mu_{l} d_{0} U_{d}^{2}$ [refer to Eq. (24)]. The nondimensional average droplet velocity $U_{d}{ }^{*}=\frac{U_{d}}{U_{c}}$, whereas the nondimensional contact line velocity is $U_{c l}{ }^{*}=\frac{U_{c l}}{U_{c}}$. The nondimensional rate of change of viscous dissipation is $\dot{E}_{v}^{*}=\frac{\dot{E}_{v}}{E_{c}}$, whereas the nondimensional rate of change of contact-line dissipation is $\dot{E}_{c l}^{*}=\frac{\dot{E}_{c l}}{\dot{E}_{c}}$. It is observed that the velocities $U_{d}{ }^{*}$ and $U_{c l}{ }^{*}$ are of the same order [see inset of Fig. 7(a)] and hence we can write

$$
\frac{\dot{E}_{c l}^{*}}{{\dot{E_{v}}}^{*}} \approx \frac{\mu_{f}}{\mu_{l}}
$$



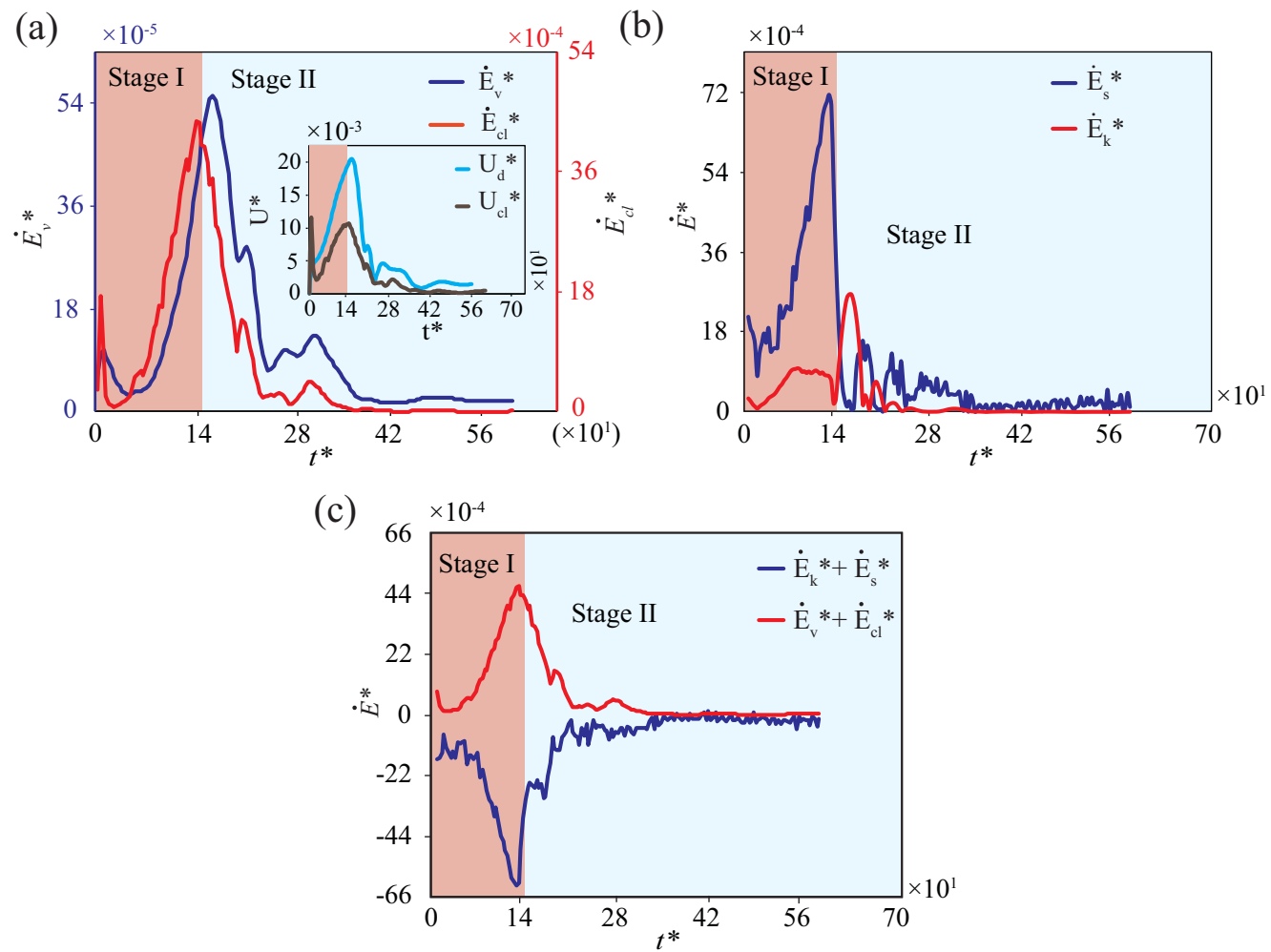

FIG. 7. (a) Variation of the nondimensional viscous and contact line dissipation rates of the droplet $\dot{E}_{v}{ }^{*}$ and ${\dot{E_{c l}}}^{*}$, respectively, with $t^{*}$. (b) Variation of the nondimensional rate of change of surface energy of the droplet $\dot{E}_{s}{ }^{*}$ and the kinetic energy of the droplet $\dot{E}_{k}{ }^{*}$ with $t^{*}$. (c) Variation of $\left(\dot{E}_{s}{ }^{*}+\dot{E}_{k}{ }^{*}\right)$ and $\left(\dot{E}_{v}{ }^{*}+\dot{E}_{c l}{ }^{*}\right)$ with $t^{*}$.

Hence, for a high-viscosity liquid droplet $\left(\mu_{l}>0.1\right), \dot{E}_{v}{ }^{*}$ dominates over $\dot{E}_{c l}{ }^{*}$. But, as in the present case, we considered a low-viscosity liquid (water droplet), so $\dot{E_{c l}}{ }^{*}$ dominates over $\dot{E}_{v}{ }^{*}$ as shown in Fig. 7(a) (note the two ordinate-axis scales differ by an order of magnitude). It is observed that both $\dot{E}_{c l}{ }^{*}$ and $\dot{E}_{v}{ }^{*}$ increases sharply in Stage I (up to $t^{*}=161$ ) because of the sharp increase in $U_{d}{ }^{*}$ and $U_{c l}{ }^{*}$ [see the inset of Fig. 7(a)]. Once the droplet is exposed to the superhydrophobic wedge and the branches of the $\mathrm{Y}$ track, both $U_{d}{ }^{*}$ and $U_{c l}{ }^{*}$ start decreasing and hence the decreasing trend of $\dot{E}_{c l}{ }^{*}$ and $\dot{E}_{v}{ }^{*}$.

Figure 7(b) shows that, during droplet transport, the nondimensional rate of change of surface energy of the droplet, $\dot{E}_{s}{ }^{*}$, dominates over the nondimensional rate of change of kinetic energy of the droplet, $\dot{E}_{k}{ }^{*}$. The surface energy of the droplet decreases continuously. The surface energy reduction rate $\dot{E}_{s}{ }^{*}$ increases sharply up to the end of Stage I because of the sharp spreading of the droplet (up to $t^{*}=161$ ). For $t^{*} \geqslant 161$ (Stage II), because of the obstruction in droplet transport by the superhydrophobic wedge, the (reduction) rate of surface energy change $\dot{E}_{s}{ }^{*}$ slows down. In Stage I, the rate of decrease in surface energy is stronger than in Stage II. This is because of the higher wettability gradient $\lambda=0.47$ in the base track compared with $\lambda=0.03$ in the branch track. In Stage I, the surface energy reduction is $\approx 60 \%$, whereas in Stage II the reduction is around $20 \%$. The total reduction in surface energy in the whole process is $\approx 80 \%$, and the remaining $\approx 20 \%$ surface energy stays in the droplet. The rate of change of kinetic energy $\dot{E}_{k}{ }^{*}$ increases up to the end of Stage I because of the increase in the droplet velocity $U_{d}$ in Stage I. This increasing trend of droplet velocity lasts up to the beginning of Stage II; after that, $\dot{E}_{k}{ }^{*}$ decreases because of the low inertia of the droplet and the low wettability gradient of the branches. 
TABLE I. Nondimensional initial and final surface energy of the droplet at Stage II, $\kappa_{i}$ and $\kappa_{f}$, respectively, for $\frac{w_{2}}{w_{1}}=1$ and $\alpha=90^{\circ}$.

\begin{tabular}{lccc}
\hline \hline Model & $\kappa_{i}$ & $\kappa_{f}$ & $\kappa_{i}-\kappa_{f}$ \\
\hline Numerical & 0.49 & 0.23 & 0.26 \\
\hline \hline
\end{tabular}

As shown in Eq. (20), the available energies are $E_{s}$ and $E_{k}$, whereas these energies dissipate in the form of $E_{v}$ and $E_{c l}$ [40]. Thus, as per the energy conservation, $\left(\dot{E}_{s}+\dot{E}_{k}\right)=\left(\dot{E}_{v}+\dot{E}_{c l}\right)$ [refer to Eq. (20)]. Figure 7(c) shows that, during the droplet transport $\left(\dot{E}_{s}{ }^{*}+\dot{E}_{k}{ }^{*}\right)$ and $\left(\dot{E}_{v}{ }^{*}+\dot{E}_{c l}{ }^{*}\right)$ are the mirror image of each other. At any particular $t^{*}$, the sum of all $\dot{E}^{*}$ is almost zero, thus energy is conserved during droplet transport and splitting.

A droplet can be split into any number of parts with the help of external energy. The energy required to split a droplet of radius $R$ into $n$ numbers of equal parts can be expressed as $E_{\text {req }}=$ $4 \pi R^{2} \sigma\left(n^{1 / 3}-1\right)$. For the equal branch width $\left(w_{1}=w_{2}=q\right)$, the droplet splits into two equal parts with the help of the wettability gradient on the branches and the superhydrophobic wedge. The theoretical nondimensional surface energy $\kappa=\frac{E_{\text {req }}}{E_{s o}}$ required to split a droplet into two equal parts is $0.26(n=2)$, where $E_{s o}$ denotes the initial surface energy of the droplet, which is defined in Sec. III A. The numerical values of $\kappa_{i}$ and $\kappa_{f}$ are shown in Table I. $\left(\kappa_{i}-\kappa_{f}\right)$ corresponds the energy loss during the splitting. Note that the numerical prediction of $\left(\kappa_{i}-\kappa_{f}\right)=0.26$ is in very good agreement with the theoretical prediction of nondimensional energy required to split a droplet $(\kappa=$ 0.26). We also calculated the available surface energy of the droplet just after the splitting of the droplet (end of Stage II), as shown in Appendix A 2. We defined the nondimensional final surface energy of Stage II as $\kappa_{f}=\frac{E_{s, f}}{E_{s o}}$, where the expression of $E_{s, f}$ is $\sigma\left[\left(p_{1} L_{2}\right)-\left(L_{2} q\right) \cos \left(\frac{\theta^{M}+\theta^{L}}{2}\right)\right]$ (refer to Appendix A 2). We obtained the theoretical value of $\kappa_{f}=0.27$, which is close to the numerical prediction of $\kappa_{f}=0.23$.

\section{Effect of geometrical parameters of the droplet and the $Y$ track}

In the previous sections, we mentioned that the droplet spreads continuously while it moves and splits into two equal parts. Thus, the height and wetted area of the droplet changes continuously. During droplet movement, the wetted area of the droplet steadily increases because of the continuous spreading of the droplet. Figure 8(a) shows that $A^{*}$ increases sharply in Stage I because of the sharp wettability gradient $(\lambda=0.47)$ in the base track of Y. From the end of Stage I to $t^{*}=294, A^{*}$ still increases sharply because of the inertia gained by the droplet in Stage I. At approximately $t^{*}=294$ the droplet loses the whole inertia gained from Stage I. For $t^{*} \geqslant 294$, most of the droplet is on the branches of the Y track, and since the branches have low wettability gradient $(\lambda=0.03)$, the droplet spreads slowly and hence the increase in $A^{*}$ slows down.

We defined the nondimensional height of the droplet as $h^{*}=h / L$. The symbol $h$ denotes the maximum height of the droplet, and $L=\left(L_{1}+L_{2}\right)$ is the total physical length of the wettabilitygradient track. Figure 8(b) shows that $h^{*}$ decreases sharply in Stage I because of the sharp spreading of the droplet on the base track of Y. In Stage II also $h^{*}$ decreases, however, the rate of decrease in $h^{*}$ slows down. This is because of the low wettability gradient $(\lambda=0.03)$ in the branches of the Y track (length $L_{2}$ ) compared with the base track (length $L_{1}$ ). $h^{*}$ decreases by almost $60 \%$ in Stage I and $\approx 20 \%$ in Stage II.

Figure 8(c) shows that the nondimensional time $t_{s}{ }^{*}$ taken by the droplet to split into two parts is decreasing as we increase the superhydrophobic wedge angle $\alpha$ up to $90^{\circ}$. Upon further increase in $\alpha, t_{s}^{*}$ increases. Thus, $\alpha=90^{\circ}$ is the optimum superhydrophobic wedge angle at the bifurcation point of the $\mathrm{Y}$ track. The inset of Fig. 8(c) shows the geometrical parameters of the superhydrophobic wedge. The length of $L_{0}=L_{2} \sin \frac{\alpha}{2}$ can be considered as the projected obstruction length by the superhydrophobic wedge on the droplet. For a large value of $\alpha$ (close to 

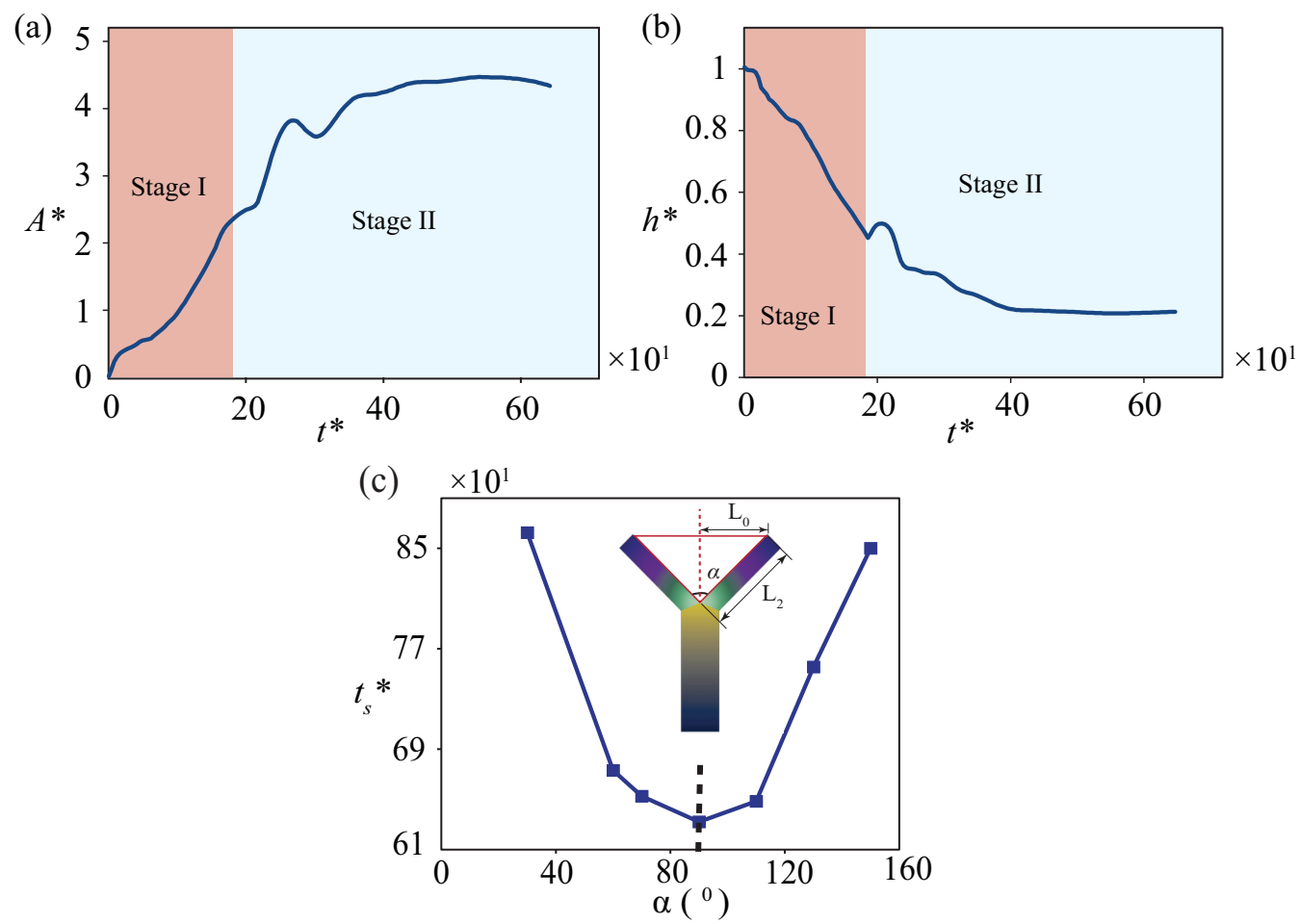

FIG. 8. (a) Variation of the nondimensional wetted area of the droplet $A^{*}$ and (b) variation of the nondimensional height of the droplet $h^{*}$ are plotted as a function of nondimensional time $t^{*}$ while the droplet transport and split on the $\mathrm{Y}$ track with superhydrophobic wedge angle $\alpha=90^{\circ}$ at the bifurcation point. (c) Nondimensional splitting time $t_{s}{ }^{*}$ taken by the droplet to split into two parts is plotted for different superhydrophobic wedge angles $\alpha$.

$180^{\circ}$ ), $L_{0}$ is maximum (close to $L_{2}$ ) and thus the droplet is exposed to the highest obstruction, so the droplet takes a higher $t_{s}{ }^{*}$ to split. Upon decreasing the value of $\alpha$ (up to $90^{\circ}$ ), the droplet is exposed to a lower $L_{0}$ and thus $t_{s}^{*}$ decreases. For $\alpha \leqslant 90^{\circ}$, the superhydrophobic wedge becomes narrower at the bifurcation point and thus some parts of the droplet are on the superhydrophobic wedge (refer to Appendix A 1). We defined a parameter called the nondimensional attachment area at the bifurcation point, $A^{*}=A / A_{0}$, where $A$ is the attachment area of the two branching fluids at the bifurcation point and $A_{0}=\pi d_{0}^{2} / 4$ is the initial projected area of the droplet. For $\alpha=90^{\circ}, A^{*}$ is 0.167 . Upon further decreasing the value of $\alpha$ (less than $90^{\circ}$ ), $A^{*}$ starts increasing because of the sharpening of the superhydrophobic wedge at the bifurcation point. For $\alpha=30^{\circ}, A^{*}$ increases to 0.257 , which leads to an additional attachment force $F_{a}$ between the two halves of the droplet in the bifurcation region (refer to Appendix A 1) as compared with $\alpha=90^{\circ}$. Thus, the droplet takes a longer time (higher $t_{s}{ }^{*}$ ) to split as compared with $\alpha=90^{\circ}$. Figure 8(c) shows that a decreasing value of $\alpha$ (below $90^{\circ}$ ) leads to an increase in $t_{s}{ }^{*}$.

\section{Three different regimes in the droplet-splitting stage (Stage II)}

At the end of the Stage I, the droplet is exposed to the superhydrophobic wedge and thus the droplet loses its inertia. It is observed from the numerical results that the kinetic energy of the droplet at Stage II is two to three orders of magnitude less than the surface energy of the droplet. We identified three different regimes in Stage II depending on the movement of the droplet front on 


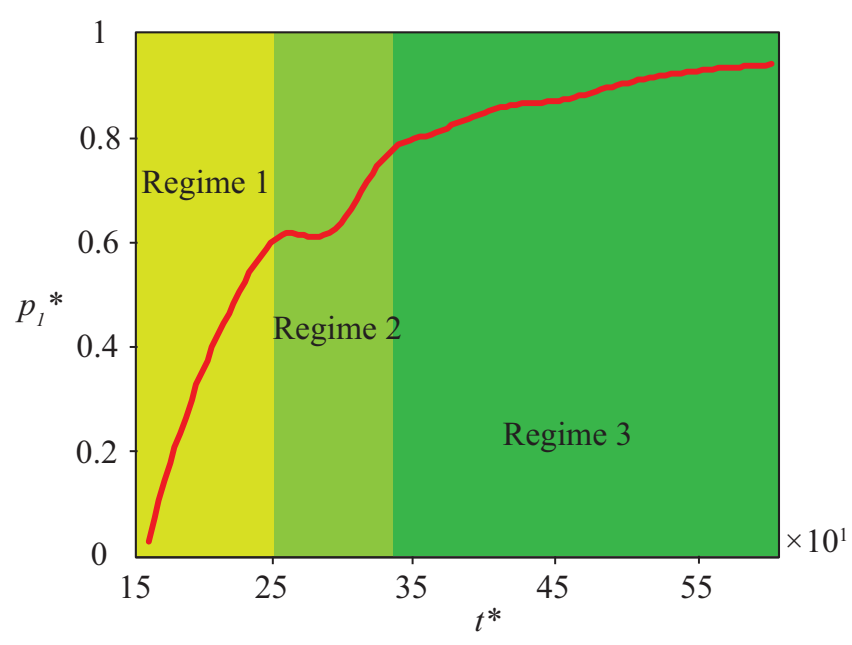

FIG. 9. The three distinct regimes during the droplet-splitting stage (Stage II). In Regime $1, p_{1}{ }^{*}=0.01 t^{*}-$ 0.92 with $R^{2}=0.98$, whereas in Regime $3, p_{1}{ }^{*}=0.78 t^{* 1 / 7}-1$ with $R^{2}=0.98$.

the branches, as shown in Fig. 9. In Regime 1, the capillary force drives the droplet and the resistance force in the bulk and the contact line of the droplet resist the droplet motion. We defined a parameter called the nondimensional front position of the droplet in branch as $p_{1}{ }^{*}=\frac{x_{1}}{L_{2}}$, where $x_{1}$ is the droplet front position along the direction of droplet spreading in the branch. The component of capillary force in the direction of droplet spreading $\left(x_{1}\right)$ is $F_{c} \sim \sigma q\left(\cos \theta^{L}-\cos \theta^{M}\right)$. The resistance force on the droplet is of the form of hydrodynamic resistance which is at the bulk of the droplet and the contact line resistance. From the above discussion in Sec. IV B, the hydrodynamic resistance is $F_{v} \sim \mu_{l} d_{0} U_{d}$, whereas the resistance at the contact line is $F_{c l} \sim \mu_{f} d_{0} U_{c l}$. Both $U_{d}$ and $U_{c l}$ are of the same order of magnitude and scale as $\approx \frac{x_{1}}{t}$, thus the total resistance on the droplet is $F_{r}=$ $F_{v}+F_{c l}=\left(\mu_{l}+\mu_{f}\right) d_{0} \frac{x_{1}}{t}$. As in Regime 1, the droplet moves due to the balance of $F_{c}$ and $F_{r}$, so we can write

$$
\begin{gathered}
x_{1} \sim t, \\
p_{1}{ }^{*} \sim \frac{\mu_{l}\left(L_{1}+L_{2}\right)}{\sigma L_{2}\left|\cos \theta^{H}-\cos \theta^{L}\right|} t^{*} .
\end{gathered}
$$

The dependency of the nondimensional front position $p_{1}{ }^{*}$ with the nondimensional time $t^{*}$ is $p_{1}{ }^{*} \sim$ $t^{*}$, which is also observed in our numerical results with $R^{2}=0.98$, as shown in Regime 1 of Fig. 9 . We identified that the droplet front spreads on the branch slowly (Tanner's spreading) in Regime 3. The liquid movement in this regime is governed by the dissipation of energy [44]. The viscous dissipation is in the bulk liquid which occurs through the rolling motion on the liquid surface. During this motion, the liquid packets from the free surface comes into the contact line (liquid front). For such flow, assuming the lubrication approximation, the velocity (Poiseuille-flow assumption) of the liquid front on the branch can be predicted as $u \sim \frac{e^{2} \Delta p}{\mu_{l} x_{1}}$, where $e$ is the height of the liquid film in the branch and $\Delta p$ is the Laplace pressure gradient at the liquid front. For $e \ll x_{1}$, the mean curvature of the liquid front $\approx \frac{e}{x_{1}^{2}}[44]$, and $\Delta p \sim \sigma \frac{e}{x_{1}^{2}}$. Thus,

$$
u \sim \frac{x_{1}}{t} \sim \frac{\sigma e^{3}}{\mu_{l} x_{1}^{3}}
$$




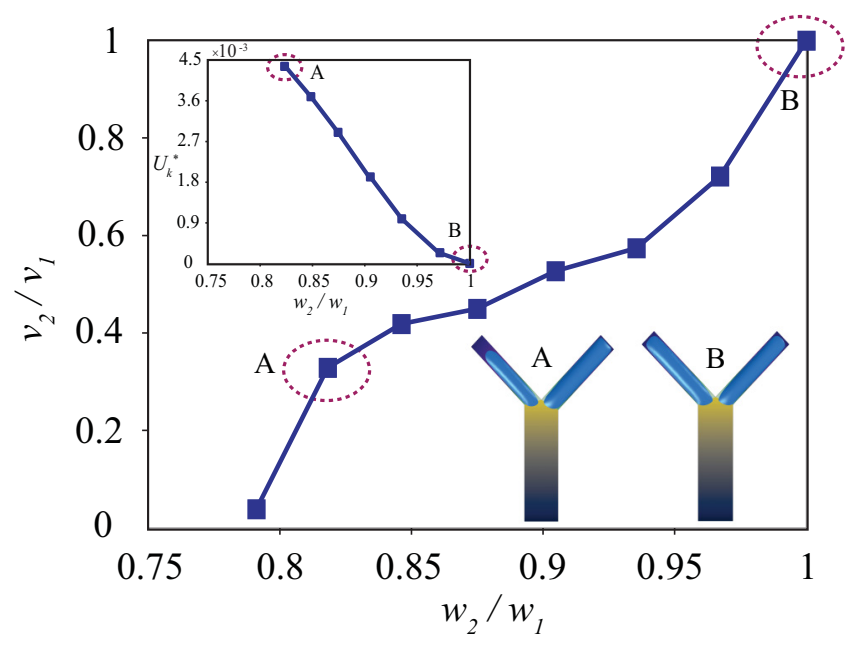

FIG. 10. The splitting volume ratios of the droplet $v_{2} / v_{1}$ is plotted with the branch-width ratios of the $\mathrm{Y}$ track $w_{2} / w_{1}$ for the superhydrophobic wedge angle $\alpha=90^{\circ}$.

The liquid height $e$ on the branches changes with time and, as the volume of the droplet $V$ is distributed equally on each branch for equal branch width $\left(w_{1}=w_{2}=q\right)$, we can write $e=\frac{V}{2 q x_{1}}$. Thus, Eq. (31) can be expressed as

$$
\begin{gathered}
x_{1} \sim\left(\frac{\sigma V^{3}}{8 \mu_{l} q^{3}}\right)^{1 / 7} t^{1 / 7}, \\
p_{1}^{*} \sim\left(\frac{V}{2 q}\right)^{3 / 7} \frac{\left(L_{1}+L_{2}\right)^{1 / 7}}{L_{2}\left|\cos \theta^{H}-\cos \theta^{L}\right|^{1 / 7}} t^{* 1 / 7} .
\end{gathered}
$$

From the above equation we find that $p_{1}{ }^{*}$ obeys the relationship with $t^{*}$ as $p_{1}{ }^{*} \sim t^{* 1 / 7}$ in Regime 3. From Fig. 9, we obtained $p_{1}{ }^{*} \sim t^{* 1 / 7}$ with $R^{2}=0.98$. The theoretical prediction is in good agreement with our numerical results. We call Regime 2 the transition regime, where the droplet front movement changes from the capillary-viscous regime to the Tanner's spreading regime.

\section{E. Effect of branch-width ratios on droplet splitting}

The droplet-splitting parameter changes significantly for different configuration of the $\mathrm{Y}$ track. As shown in Fig. 10, for branch widths $w_{1}=w_{2}$, the droplet splits into two halves $\left(v_{1}=v_{2}\right)$. For an unequal width of the branches of the $\mathrm{Y}$ track $\left(w_{1} \neq w_{2}\right)$, the droplet splits into unequal volumes $\left(v_{1} \neq v_{2}\right)$. Figure 10 shows that, as $w_{2} / w_{1}$ increases, $v_{2} / v_{1}$ increases. An increase in $w_{2} / w_{1}$ means an increase in the branch width $w_{2}$ and a decrease in the branch width $w_{1}$. A higher branch width can accumulate more volume. Thus, upon increasing $w_{2}$, the volume $v_{2}$ (volume corresponds to the branch width $w_{2}$ ) increases which leads to an increase in $v_{2} / v_{1}$. We defined a parameter $U_{k}{ }^{*}$ which is the difference in average $U^{*}$ between two branching fluids at $t^{*}=320$ (Stage II) where the division of two branching fluid is made by cutting the droplet through the bifurcation point. The variation of $v_{2} / v_{1}$ is nonlinear with $w_{2} / w_{1}$ from point $\mathrm{A}$ to point $\mathrm{B}$. This is because of the nonlinearity of $U_{k}{ }^{*}$ at a particular $t^{*}$ of Stage II, with $w_{2} / w_{1}$ as shown in the inset of Fig. 10. For $w_{2} / w_{1} \leqslant 0.79$, the droplet cannot split into two parts, which is explained in the next section. We consider that $w_{2} / w_{1}=0.79$ is the critical branch-width ratio. A droplet can be split into the desired volume by manipulating the branch width of the Y track. 


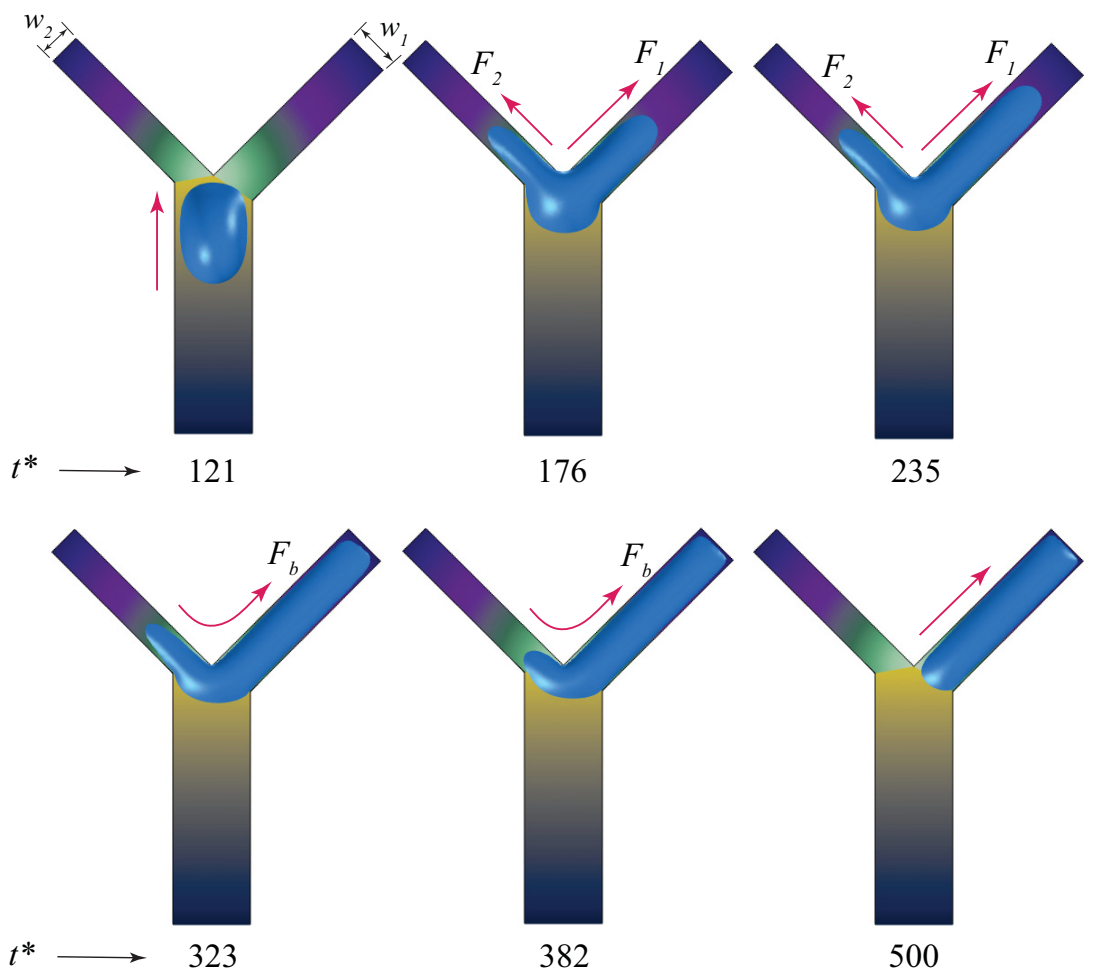

FIG. 11. Droplet dynamics for width ratio $w_{2} / w_{1}=0.714$ and the superhydrophobic wedge angle $\alpha=90^{\circ}$.

\section{F. Dynamics of the droplet for $w_{2} / w_{1} \leqslant 0.79$ and $\alpha=90^{\circ}$}

For the branch-width ratio $w_{2} / w_{1} \leqslant 0.79$, in Stage II, although initially the droplet starts splitting into two branches but because of the dominance of driving Laplace pressure towards the higher branch-width side, the droplet finally enters into the higher-width branch, as shown in Fig. 11. Force exerted on the droplet due to the Laplace pressure is scaled as $F_{b} \sim \sigma\left(\frac{2}{w_{1}}-\frac{2}{w_{2}}\right) w_{1} L_{2}$, where the radius of curvature at the droplet front and back is taken as $w_{1} / 2$ and $w_{2} / 2$, respectively. As the droplet becomes a film in the branch and thus the other two radii of curvature at the droplet front and back in both branches are $\gg w_{1} / 2$ and $w_{2} / 2$ and thus it does not contribute in $F_{b}$. The forces due to the wettability gradient on both branches of widths $w_{1}$ and $w_{2}$ is scaled as $F_{1} \sim$ $\sigma w_{1}\left(\cos \theta^{M}-\cos \theta^{L}\right)$ and $F_{2} \sim \sigma w_{2}\left(\cos \theta^{M}-\cos \theta^{L}\right)$, respectively. The hydrodynamic resistance force on the droplet can be scaled as $F_{R} \sim \frac{\mu_{l} u_{1} w_{1} L_{2}}{e}$, which is two to three orders of magnitude less than $F_{b}, F_{1}$, and $F_{2}$. In the initial phase of Stage II (from $t^{*}=121$ to $t^{*}=235$ ), the droplet spreads on the branches due to the wettability gradient on the branches and also due to the inertia gained by the droplet in Stage I. During that, the spreading is more on branch 2 as compared with the branch 1 , as shown in Fig. 11. This is because of the higher driving force $\left(F_{2}>F_{1}\right)$ on branch 2 . This phenomena of unequal-droplet spreading happens until the inertia of the droplet vanishes completely (until $t^{*}=235$ ). For $t^{*}>235$, as the inertia of the droplet is close to zero, the competition between $F_{b}, F_{1}$, and $F_{2}$ drives the droplet. It is observed that the scaled values of $\frac{F_{b}}{F_{1}}$ and $\frac{F_{b}}{F_{2}}$ are $\approx 30$ and $\approx 40$, respectively, for $w_{2} / w_{1}=0.714$. Thus, $F_{b}$ dominates over $F_{1}$ and $F_{2}$ and the direction of $F_{b}$ is towards branch 2, so the droplet moves towards the higher branch width side and settles there, as shown in the Fig. 11. Considering the Poiseuille flow of the droplet due to the driving Laplacepressure force $F_{b}$, the velocity of the droplet is $u_{1}=\frac{e^{2} \Delta p}{12 \mu_{l} L_{2}}$, where $e$ is the height of the droplet which can be approximated as $e \sim \frac{V}{L_{2}\left(w_{1}+w_{2}\right)}$ and $\Delta p=\sigma\left(\frac{2}{w_{1}}-\frac{2}{w_{2}}\right)$ is the driving Laplace pressure. 
Thus, the nondimensional velocity of the droplet $u_{1}{ }^{*}$ becomes

$$
u_{1}^{*} \sim \frac{\sigma\left(\frac{2}{w_{1}}-\frac{2}{w_{2}}\right) V^{2}}{24 \mu_{l} L_{2}^{3}\left(w_{1}+w_{2}\right)^{2} U_{c}} .
$$

From the above equation, the theoretical prediction of $u_{1}{ }^{*}$ is 0.001 which matches well with our numerical prediction $\left(u_{1}{ }^{*}=0.0014\right)$ for $w_{2} / w_{1}=0.714$ and $\alpha=90^{\circ}$.

\section{CONCLUSION}

We studied passive droplet transport and splitting on an open surface using a Y-shaped wettability-gradient track on the surface. Generally, microchannels are widely used in microfluidic devices for droplet mixing, metering, and splitting; however, the fabrication of microchannels is quite challenging and time-consuming. An open surface droplet manipulation gives a better alternative. The Y-shaped wettability-gradient track with the superhydrophobic background is used for wettability patterning of an open surface in the present work. A droplet initially placed at the inlet of the base track moves towards the Y junction and splits into the two branches due to the wettability gradient. The net driving force due to the wettability gradient of the Y track, in the direction of the droplet transport, is sufficient to overcome the droplet's interfacial energy to split into two parts by a superhydrophobic wedge at the bifurcation point of the Y track. The splitting of the droplet is due to the combined effect of the wettability gradient and the superhydrophobic wedge. The droplet splitting depends on the configuration of the Y track. We identified an optimum configuration (superhydrophobic wedge angle $\alpha=90^{\circ}$ ) of the Y track at which the droplet splits faster. We observed that the theoretical nondimensional energy $(\kappa=0.26)$ required to split a droplet into two equal parts matches well with the present energy loss during the splitting of the droplet $\left(\kappa_{i}-\kappa_{f}=0.26\right)$. In the proposed wettability patterning, it is possible to have symmetric and asymmetric droplet splitting by manipulating the branch widths. The droplet splits into two halves for equal branch width, whereas an unequal branch width gives an asymmetric splitting. We also identified a critical branch-width ratio $\left(\frac{w_{2}}{w_{1}}=0.79\right)$, below which the droplet does not split and moves towards the higher branch width side $\left(w_{1}\right)$ due to the back Laplace pressure. We calculated the nondimensional velocity of the droplet $u_{1}{ }^{*}=0.0014$ due to the back Laplace pressure and it matches well with the theoretical prediction of $u_{1}{ }^{*}$ for $\frac{w_{2}}{w_{1}}=0.714$ and $\alpha=90^{\circ}$. Thus, a droplet of fixed volume can be displaced by a certain distance and then divided into two desired volumes by manipulating the branch widths of the $\mathrm{Y}$ track, which has potential applications in the Lab-on-a-Chip (LOC), smart microelectronics, point-of-care (POC) diagnostics, etc. We identified three distinct regimes of droplet splitting and the temporal variations of the droplet front position in the branches through scaling arguments. We also explored the flow pattern inside the droplet during the transport and splitting. The physics of the droplet transport and splitting mechanisms is explained through scaling and with the help of the energy conservation arguments. To validate this work with experiment, one needs to create a Y-shaped wettability-gradient track of very low hysteresis [45] on a superhydrophobic background. The droplet shapes during the transport, droplet-splitting ratios for different branch width ratios, and the fluid motion inside the droplet can be matched with the corresponding experiment.

\section{ACKNOWLEDGMENTS}

We acknowledge the Scheme for Promotion of Academic and Research Collaboration (SPARC) program (Project: P644) from the Ministry of Human Resource Development, Government of India and the associated UKIERI support. M.K.T. also acknowledges support by the Wellcome/EPSRC Centre for Interventional and Surgical Sciences (WEISS) (203145Z/16/Z), Wolfson Foundation and Royal Society for his Royal Society Wolfson Fellowship, and the NICEDROPS project supported by the European Research Council (ERC) under the European Union's Horizon 2020 research and innovation programme under grant agreement No. 714712. 
(a)

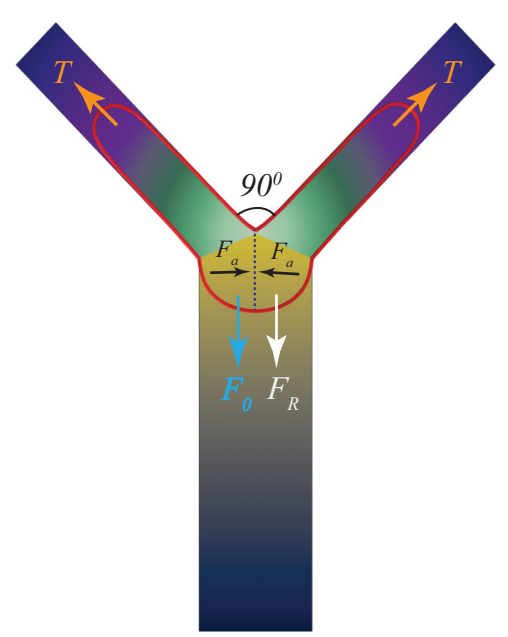

(b)

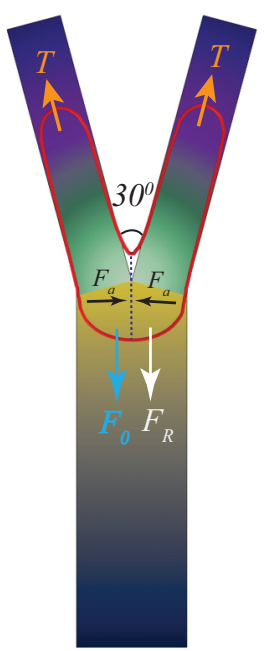

FIG. 12. Schematic of forces involved during the splitting of the droplet for $\frac{w_{2}}{w_{1}}=1$ and (a) $\alpha=90^{\circ}$, (b) $\alpha=30^{\circ}$. Red line represents the droplet contact line. The dotted line corresponds the attachment area between two parts of the droplet.

\section{APPENDIX: FORCE BALANCE AND SURFACE-ENERGY CALCULATION}

\section{Forces involved during the splitting of the droplet}

The droplet splits into two parts due to the combined effect of the wettability gradient and the superhydrophobic wedge. Figures 12(a) and 12(b) show the force balance at the bifurcation point while the droplet splits at $t^{*}=283$. We have not considered the hysteresis force in our numerical modeling. The force $T$ corresponds to the tensions on both the branches due to the wettability gradient on the branches. The force $F_{R}$ is the overall hydrodynamic resistance force. There is an obstruction force $F_{0}$ due to the superhydrophobic wedge in the opposite direction of droplet transport. The force $F_{a}$ is the cohesive force between two parts of the droplet. The cohesive force is the collective intermolecular forces such as $\mathrm{H}$ bonding and van der Waals forces. For $\alpha=30^{\circ}$, the droplet crosses the border of the superhydrophobic wedge due to the wedge's narrowness. Thus, the attachment area between two parts of the droplet is more than $\alpha=90^{\circ}$. A larger attachment area at the bifurcation junction for $\alpha=30^{\circ}$ leads to a greater attachment force $F_{a}$. The force balance at the bifurcation point is

$$
\begin{aligned}
2 T \cos \frac{\alpha}{2} & =F_{0}+F_{R}, \\
F_{a} & =F_{a} .
\end{aligned}
$$

\section{Final surface energy of the droplet in the droplet-splitting stage (Stage II)}

In the droplet-splitting stage (Stage II), the kinetic energy of the droplet is two to three orders of magnitude less than the surface energy of the droplet, $E_{s}$, and hence the total available energy of the droplet is equivalent to $E_{s}$.

For equal branch width $\left(w_{1}=w_{2}=q\right)$, the droplet splits into equal parts. As the droplets in both the branches are identical, the surface energy $E_{s}$ at the end of the splitting stage (Stage II) is 
(a)

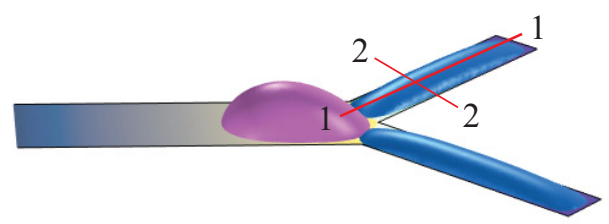

(c)

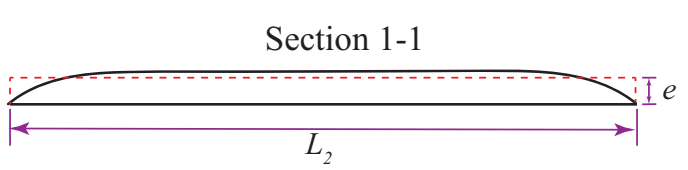

(b)

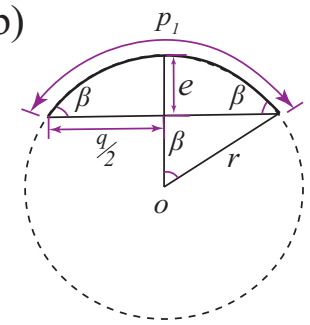

Section 2-2

FIG. 13. (a) Schematic of droplet initial and final shapes of droplet-splitting process for $\frac{w_{2}}{w_{1}}=1$ and $\alpha=$ $90^{\circ}$. (b) Corresponding cross section 2-2 of the branch fluid. (c) Corresponding section 1-1 of the branch fluid.

calculated for single branch as shown in Fig. 13(a). The cross-section view of the branch fluid is shown in Fig. 13(b). Using the geometry in Fig. 13(b), we can write

$$
\begin{gathered}
\cos \beta=\frac{r-e}{r}, \\
e=r(1-\cos \beta), \\
\tan \beta=\frac{q / 2}{r-r(1-\cos \beta)},
\end{gathered}
$$

where $e$ is the height of the droplet in branch, $q$ is the width of the branch, $r$ is the radius of curvature, and $\beta$ is the angle made by the droplet due to the confinement of the branch by the superhydrophobic region. Let us consider the droplet volume as $V$ and $L_{2}$ as the length of the branch. As the droplet splits into two halves, volume conservation gives

$$
\frac{V}{2}=\left[\frac{\beta \pi}{180} r^{2}-(r-e) \frac{q}{2}\right] L_{2} .
$$

Thus, we can calculate $r, \beta$, and $e$ by using Eqs. (A4)-(A6) for a known $V$ and $L_{2}$. Let $p_{1}$ be the total length of the droplet cap in the cross-sectional view [refer to Fig. 13(b)] which is equivalent to $2 \beta r$. The estimated $E_{s}$ of a single branch fluid is

$$
E_{s 1, f}=\sigma\left[\left(p_{1} L_{2}\right)-\left(L_{2} q\right) \cos \left(\frac{\theta^{M}+\theta^{L}}{2}\right)\right] .
$$

The total surface energy of the droplet at the end of Stage II is $E_{s, f}=2 E_{s 1, f}$.

[1] S. Haeberle and R. Zengerle, Microfluidic platforms for lab-on-a-chip applications, Lab Chip 7, 1094 (2007).

[2] J. M. Morrissette, P. S. Mahapatra, A. Ghosh, R. Ganguly, and C. M. Megaridis, Rapid, self-driven liquid mixing on open-surface microfluidic platforms, Sci. Rep. 7, 1800 (2017).

[3] C. D. Chin, V. Linder, and S. K. Sia, Commercialization of microfluidic point-of-care diagnostic devices, Lab Chip 12, 2118 (2012).

[4] U. Sen, S. Chatterjee, P. S. Mahapatra, R. Ganguly, R. Dodge, L. Yu, and C. M. Megaridis, Surfacewettability patterning for distributing high-momentum water jets on porous polymeric substrates, ACS Appl. Mater. Interfaces 10, 5038 (2018). 
[5] S. Chatterjee, P. S. Mahapatra, A. Ibrahim, R. Ganguly, L. Yu, R. Dodge, and C. M. Megaridis, Precise liquid transport on and through thin porous materials, Langmuir 34, 2865 (2018).

[6] X. Yao, Y. Song, and L. Jiang, Applications of bio-inspired special wettable surfaces, Adv. Mater. 23, 719 (2011).

[7] J. Li and Z. Guo, Spontaneous directional transportations of water droplets on surfaces driven by gradient structures, Nanoscale 10, 13814 (2018).

[8] A. Russo, M. Icardi, M. Elsharkawy, D. Ceglia, P. Asinari, and C. M. Megaridis, Numerical simulation of droplet impact on wettability-patterned surfaces, Phys. Rev. Fluids 5, 074002 (2020).

[9] M. Costalonga and P. Brunet, Directional motion of vibrated sessile drops: A quantitative study, Phys. Rev. Fluids 5, 023601 (2020).

[10] T. M. Thomas, I. U. Chowdhury, K. Dhivyaraja, P. S. Mahapatra, A. Pattamatta, and M. K. Tiwari, Droplet dynamics on a wettability patterned surface during spray impact, Processes 9, 555 (2021).

[11] Z. Yuan, M. Matsumoto, and R. Kurose, Directional migration of an impinging droplet on a surface with wettability difference, Phys. Rev. Fluids 5, 113605 (2020).

[12] N. Moumen, R. S. Subramanian, and J. B. McLaughlin, Experiments on the motion of drops on a horizontal solid surface due to a wettability gradient, Langmuir 22, 2682 (2006).

[13] J.-J. Huang, H. Huang, and X. Wang, Numerical study of drop motion on a surface with stepwise wettability gradient and contact angle hysteresis, Phys. Fluids 26, 062101 (2014).

[14] I. U. Chowdhury, P. S. Mahapatra, and A. K. Sen, Shape evolution of drops on surfaces of different wettability gradients, Chem. Eng. Sci. 229, 116136 (2020).

[15] I. U. Chowdhury, P. S. Mahapatra, and A. K. Sen, Self-driven droplet transport: Effect of wettability gradient and confinement, Phys. Fluids 31, 042111 (2019).

[16] C. Liu, J. Sun, J. Li, C. Xiang, L. Che, Z. Wang, and X. Zhou, Long-range spontaneous droplet selfpropulsion on wettability gradient surfaces, Sci. Rep. 7, 7552 (2017).

[17] F. Y. Ushikubo, F. S. Birribilli, D. R. B. Oliveira, and R. L. Cunha, Y- and t-junction microfluidic devices: Effect of fluids and interface properties and operating conditions, Microfluid. Nanofluid. 17, 711 (2014).

[18] A. Carlson, M. Do-Quang, and G. Amberg, Droplet dynamics in a bifurcating channel, Int. J. Multiphase Flow 36, 397 (2010).

[19] D. R. Link, E. Grasland-Mongrain, A. Duri, F. Sarrazin, Z. Cheng, G. Cristobal, M. Marquez, and D. A. Weitz, Electric control of droplets in microfluidic devices, Angew. Chem., Int. Ed. 45, 2556 (2006).

[20] N. J. B. Nikapitiya, M. M. Nahar, and H. Moon, Accurate, consistent, and fast droplet splitting and dispensing in electrowetting on dielectric digital microfluidics, Micro Nano Syst. Lett. 5, 24 (2017).

[21] Y. Guan, B. Li, and L. Xing, Numerical investigation of electrowetting-based droplet splitting in closed digital microfluidic system: Dynamics, mode, and satellite droplet, Phys. Fluids 30, 112001 (2018).

[22] J. Park, J. H. Jung, K. Park, G. Destgeer, H. Ahmed, R. Ahmad, and H. J. Sung, On-demand acoustic droplet splitting and steering in a disposable microfluidic chip, Lab Chip 18, 422 (2018).

[23] J. H. Jung, G. Destgeer, B. Ha, J. Park, and H. J. Sung, On-demand droplet splitting using surface acoustic waves, Lab Chip 16, 3235 (2016).

[24] Y.-F. Yap, S.-H. Tan, N.-T. Nguyen, S. S. Murshed, T.-N. Wong, and L. Yobas, Thermally mediated control of liquid microdroplets at a bifurcation, J. Phys. D: Appl. Phys. 42, 065503 (2009).

[25] Z. Long, A. M. Shetty, M. J. Solomon, and R. G. Larson, Fundamentals of magnet-actuated droplet manipulation on an open hydrophobic surface, Lab Chip 9, 1567 (2009).

[26] U. Banerjee and A. K. Sen, Shape evolution and splitting of ferrofluid droplets on a hydrophobic surface in the presence of a magnetic field, Soft Matter 14, 2915 (2018).

[27] E. Samiei and M. Hoorfar, Systematic analysis of geometrical based unequal-droplet splitting in digital microfluidics, J. Micromech. Microeng. 25, 055008 (2015).

[28] D. Song, B. Song, H. Hu, X. Du, and F. Zhou, Selectively splitting a droplet using superhydrophobic stripes on hydrophilic surfaces, Phys. Chem. Chem. Phys. 17, 13800 (2015).

[29] S. B. Berry, J. J. Lee, J. Berthier, E. Berthier, and A. B. Theberge, Droplet incubation and splitting in open microfluidic channels, Anal. Methods 11, 4528 (2019).

[30] M. K. Chaudhury and G. M. Whitesides, How to make water run uphill, Science 256, 1539 (1992). 
[31] G. Graeber, K. Regulagadda, P. Hodel, C. Küttel, D. Landolf, T. M. Schutzius, and D. Poulikakos, Leidenfrost droplet trampolining, Nat. Commun. 12, 1727 (2021).

[32] J. T. Pham, M. Paven, S. Wooh, T. Kajiya, H.-J. Butt, and D. Vollmer, Spontaneous jumping, bouncing and trampolining of hydrogel drops on a heated plate, Nat. Commun. 8, 905 (2017).

[33] Y. Li, C. Diddens, A. Prosperetti, K. L. Chong, X. Zhang, and D. Lohse, Bouncing Oil Droplet in a Stratified Liquid and its Sudden Death, Phys. Rev. Lett. 122, 154502 (2019).

[34] C. Multiphysics, Comsol Multiphysics CFD Module User Guide (Version 5.3), COMSOL, 303 (2017).

[35] X. Cai, H. Marschall, M. Wörner, and O. Deutschmann, Numerical simulation of wetting phenomena with a phase-field method using openfoam ${ }^{\circledR}$, Chem. Eng. Technol. 38, 1985 (2015).

[36] F. Bai, X. He, X. Yang, R. Zhou, and C. Wang, Three dimensional phase-field investigation of droplet formation in microfluidic flow focusing devices with experimental validation, Int. J. Multiphase Flow 93, 130 (2017).

[37] H. Liu and Y. Zhang, Droplet formation in a T-shaped microfluidic junction, J. Appl. Phys. 106, 034906 (2009).

[38] Y. Wu, F. Wang, M. Selzer, and B. Nestler, Investigation of equilibrium droplet shapes on chemically striped patterned surfaces using phase-field method, Langmuir 35, 8500 (2019).

[39] S. Daniel, S. Sircar, J. Gliem, and M. K. Chaudhury, Ratcheting motion of liquid drops on gradient surfaces, Langmuir 20, 4085 (2004).

[40] P. Attané, F. Girard, and V. Morin, An energy balance approach of the dynamics of drop impact on a solid surface, Phys. Fluids 19, 012101 (2007).

[41] Z. Li, G.-H. Hu, Z.-L. Wang, Y.-B. Ma, and Z.-W. Zhou, Three dimensional flow structures in a moving droplet on substrate: A dissipative particle dynamics study, Phys. Fluids 25, 072103 (2013).

[42] A. Carlson, G. Bellani, and G. Amberg, Contact line dissipation in short-time dynamic wetting, Europhys. Lett. 97, 44004 (2012).

[43] M. Ahmadlouydarab and J. J. Feng, Motion and coalescence of sessile drops driven by substrate wetting gradient and external flow, J. Fluid Mech. 746, 214 (2014).

[44] U. Sen, S. Chatterjee, R. Ganguly, R. Dodge, L. Yu, and C. M. Megaridis, Scaling laws in directional spreading of droplets on wettability-confined diverging tracks, Langmuir 34, 1899 (2018).

[45] G. Launay, M. S. Sadullah, G. McHale, R. Ledesma-Aguilar, H. Kusumaatmaja, and G. G. Wells, Selfpropelled droplet transport on shaped-liquid surfaces, Sci. Rep. 10, 14987 (2020). 